International Institute for Applied Systems Analysis - A-2361 Laxenburg - Austria Tel: +432236 807 • Fax: +43223671313 • E-mail: info@iiasa.ac.at • Web: www.iiasa.ac.at

INTERIM REPORT IR-97-071/October

\title{
Evolutionary Dynamics of Seed Size and Seedling Competitive Ability
}

S.A.H. Geritz (geritz@zool.umd.edu)

E. van der Meijden (meijden@rulsfb.LeidenUniv.nl)

J.A.J. Metz(metz@rulsfb.LeidenUniv.nl)

Approved by

Ulf Dieckmann (dieckman@iiasa.ac.at)

Project Coordinator, Adaptive Dynamics Network

Interim Reports on work of the International Institute for Applied Systems Analysis receive only limited review. Views or opinions expressed herein do not necessarily represent those of the Institute, its National Member Organizations, or other organizations supporting the work. 


\section{IIASA STUdies IN AdAPTIVE Dynamics}

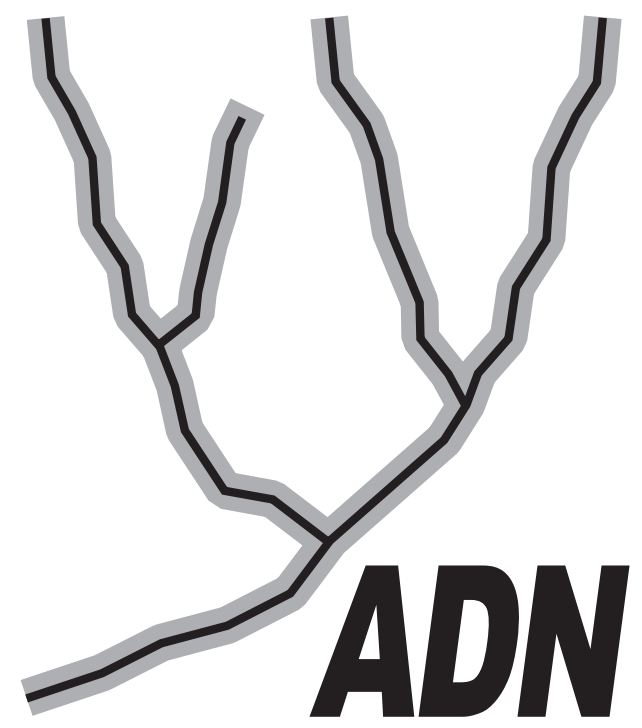

The Adaptive Dynamics Network at IIASA fosters the development of new mathematical and conceptual techniques for understanding the evolution of complex adaptive systems.

Focusing on these long-term implications of adaptive processes in systems of limited growth, the Adaptive Dynamics Network brings together scientists and institutions from around the world with IIASA acting as the central node.

Scientific progress within the network is reported in the IIASA Studies in Adaptive Dynamics series.

\section{The Adaptive Dynamics Network}

The pivotal role of evolutionary theory in life sciences derives from its capability to provide causal explanations for phenomena that are highly improbable in the physicochemical sense. Yet, until recently, many facts in biology could not be accounted for in the light of evolution. Just as physicists for a long time ignored the presence of chaos, these phenomena were basically not perceived by biologists.

Two examples illustrate this assertion. Although Darwin's publication of "The Origin of Species" sparked off the whole evolutionary revolution, oddly enough, the population genetic framework underlying the modern synthesis holds no clues to speciation events. A second illustration is the more recently appreciated issue of jump increases in biological complexity that result from the aggregation of individuals into mutualistic wholes.

These and many more problems possess a common source: the interactions of individuals are bound to change the environments these individuals live in. By closing the feedback loop in the evolutionary explanation, a new mathematical theory of the evolution of complex adaptive systems arises. It is this general theoretical option that lies at the core of the emerging field of adaptive dynamics. In consequence a major promise of adaptive dynamics studies is to elucidate the long-term effects of the interactions between ecological and evolutionary processes.

A commitment to interfacing the theory with empirical applications is necessary both for validation and for management problems. For example, empirical evidence indicates that to control pests and diseases or to achieve sustainable harvesting of renewable resources evolutionary deliberation is already crucial on the time scale of two decades.

The Adaptive Dynamics Network has as its primary objective the development of mathematical tools for the analysis of adaptive systems inside and outside the biological realm. 


\section{IIASA STUdies IN AdAPTIVE DYNAMicS}

No. 1 Metz JAJ, Geritz SAH, Meszéna G, Jacobs FJA, van Heerwaarden JS: Adaptive Dynamics: A Geometrical Study of the Consequences of Nearly Faithful Reproduction.

IIASA Working Paper WP-95-99.

van Strien SJ, Verduyn Lunel SM (eds.): Stochastic and Spatial Structures of Dynamical Systems, KNAW Verhandelingen, North Holland, Amsterdam, pp. 183-231 (1996).

No. 2 Dieckmann U, Law R:

The Dynamical Theory of Coevolution: A Derivation from Stochastic Ecological Processes.

IIASA Working Paper WP-96-01.

Journal of Mathematical Biology (1996) 34, 579-612.

No. 3 Dieckmann U, Marrow P, Law R:

Evolutionary Cycling of Predator-Prey Interactions: Population Dynamics and the Red Queen.

Journal of Theoretical Biology (1995) 176, 91-102.

No. 4 Marrow P, Dieckmann U, Law R:

Evolutionary Dynamics of Predator-Prey Systems: An Ecological Perspective.

IIASA Working Paper WP-96-02.

Journal of Mathematical Biology (1996) 34, 556-578.

No. 5 Law R, Marrow P, Dieckmann U:

On Evolution under Asymmetric Competition.

IIASA Working Paper WP-96-03.

Evolutionary Ecology (1997) 11, 485-501.

No. 6 Metz JAJ, Mylius SD, Diekmann O:

When Does Evolution Optimise? On the Relation between Types of Density Dependence and Evolutionarily Stable Life History Parameters.

IIASA Working Paper WP-96-04.

No. 7 Ferrière R, Gatto M:

Lyapunov Exponents and the Mathematics of Invasion in Oscillatory or

Chaotic Populations.

Theoretical Population Biology (1995) 48, 126-171.

No. 8 Ferrière R, Fox GA:

Chaos and Evolution.

Trends in Ecology and Evolution (1995) 10, 480-485.

No. 9 Ferrière R, Michod RE:

The Evolution of Cooperation in Spatially Heterogeneous Populations.

IIASA Working Paper WP-96-29.

American Naturalist (1996) 147, 692-717. 
No. 10 Van Dooren TJM, Metz JAJ:

Delayed Maturation in Temporally Structured Populations with Non-

Equilibrium Dynamics.

IIASA Working Paper WP-96-70.

Journal of Evolutionary Biology (1997) in press.

No. 11 Geritz SAH, Metz JAJ, Kisdi E, Meszéna G:

The Dynamics of Adaptation and Evolutionary Branching.

IIASA Working Paper WP-96-77.

Physical Review Letters (1997) 78, 2024-2027.

No. 12 Geritz SAH, Kisdi E, Meszéna G, Metz JAJ:

Evolutionarily Singular Strategies and the Adaptive Growth and Branching of the Evolutionary Tree.

IIASA Working Paper WP-96-114.

Evolutionary Ecology (1997) in press.

No. 13 Heino M, Metz JAJ, Kaitala V:

Evolution of Mixed Maturation Strategies in Semelparous Life-Histories:

the Crucial Role of Dimensionality of Feedback Environment.

IIASA Working Paper WP-96-126.

Philosophical Transactions of the Royal Society of London Series B (1997) in press.

No. 14 Dieckmann U:

Can Adaptive Dynamics Invade?

IIASA Working Paper WP-96-152.

Trends in Ecology and Evolution (1997) 12, 128-131.

No. 15 Meszéna G, Czibula I, Geritz SAH:

Adaptive Dynamics in a Two-Patch Environment: a Simple Model for Allopatric and Parapatric Speciation.

IIASA Interim Report IR-97-01.

Journal of Biological Systems (1997) in press.

No. 16 Heino M, Metz JAJ, Kaitala V:

The Enigma of Frequency-Dependent Selection.

IIASA Interim Report IR-97-61.

No. 17 Heino M:

Management of Evolving Fish Stocks.

IIASA Interim Report IR-97-62.

No. 18 Heino M:

Evolution of Mixed Reproductive Strategies in Simple Life-History Models. IIASA Interim Report IR-97-63.

No. 19 Geritz SAH, van der Meijden E, Metz JAJ:

Evolutionary Dynamics of Seed Size and Seedling Competitive Ability. IIASA Interim Report IR-97-71.

No. 20 Galis F, Metz JAJ:

Why are there so many Cichlid Species? On the Interplay of Speciation and Adaptive Radiation.

IIASA Interim Report IR-97-72.

Trends in Ecology and Evolution (1997) in press. 
No. 21 Boerlijst MC, Nowak MA, Sigmund K:

Equal Pay for all Prisoners. / The Logic of Contrition.

IIASA Interim Report IR-97-73.

AMS Monthly (1997) 104, 303-307.

Theoretical Biology (1997) 185, 281-294.

No. 22 Law R, Dieckmann U:

Symbiosis without Mutualism and the Merger of Lineages in Evolution. IIASA Interim Report IR-97-74.

No. 23 Klinkhamer PGL, de Jong TJ, Metz JAJ:

Sex and Size in Cosexual Plants.

IIASA Interim Report IR-97-78.

Trends in Ecology and Evolution (1997) 12, 260-265.

Issues of the IIASA Studies in Adaptive Dynamics series can be obtained free of charge. Please contact:

Adaptive Dynamics Network

International Institute for Applied Systems Analysis

Schloßplatz 1

A-2361 Laxenburg

Austria

Telephone +432236 807, Telefax +432236 71313, E-Mail adn@iiasa.ac.at, Internet http://www.iiasa.ac.at/Research/ADN 


\section{Contents}

Introduction 1

Evolutionarily Singular Strategies $\quad 5$

Resident population dynamics and mutant's fitness 5

$\begin{array}{ll}\text { Evolutionarily stable singular strategies } & 7\end{array}$

$\begin{array}{lr}\text { Convergence stable singular strategies } & 8\end{array}$

$\begin{array}{ll}\text { Evolutionary branching } & 9\end{array}$

$\begin{array}{ll}\text { Changes in number and stability properties of evolutionary singular strategies } & 11\end{array}$

$\begin{array}{ll}\text { Evolutionarily Singular Coalitions } & 13\end{array}$

$\begin{array}{ll}\text { Resident population dynamics and mutant's fitness } & 13\end{array}$

$\begin{array}{ll}\text { Isoclines and evolutionarily singular coalitions } & 14\end{array}$

Change in number and stability properties of evolutionary singular coalitions 17

$\begin{array}{ll}\text { Generalization to higher levels of polymorphism } & 19\end{array}$

$\begin{array}{ll}\text { Extinction of evolutionary branches } & 20\end{array}$

Conclusions and Discussion $\quad 22$

Appendix 28

Isoclines and their connection to the boundary of the area of coexistence $\quad 28$

Loss of global evolutionary stability and the emergence of new singularities 29

References 31 


\begin{abstract}
We present a model for the evolutionary dynamics of seed size when seedlings from large seeds are better competitors than seedlings from small seeds and there is a tradeoff between seed size and seed number. We first consider two limiting cases where seed size has either no effect on the competitive ability of seedlings, or where seedlings from larger seeds always win from seedlings from smaller seeds if together in the same germination site. In the first case there is a single evolutionary optimal seed size excluding all other, whereas in the second case there is an evolutionarily stable seed polymorphism with a continuous variation of seed sizes where plants with small (but numerous seeds) survive by exploiting sites that by chance remain unoccupied by plants with larger (but less numerous) seeds. We investigate how these two cases connect to one another via intermediate levels of competitive asymmetry. We find that strong competitive asymmetry and high resource levels favor coexistence of plants with different seed sizes when seed and seedling survival is moderately low but large seeds have a substantial precompetitive advantage over smaller seeds. Assuming mutationlimited evolution and assuming that single mutations have only a small phenotypic effect, an initially monomorphic population with a single seed size will reach the final evolutionarily stable polymorphic state through a series of discrete evolutionary branching events. At each branching event, a given lineage already present in the population divides into two daughter lines, each with its own seed size. If precompetitive seed and seedling survival is high for small and large seeds alike, evolutionary branching may be followed by extinction of one or more lineages (including mass-extinction), and thus not necessarily gives rise to evolutionarily stable seed polymorphisms. Various results presented here are model-independent and point the way to a more general evolutionary bifurcation theory describing how the number and stability properties of evolutionary equilibria can change as a consequence of changes in model parameters.
\end{abstract}

Keywords: Seed size evolution, seedling competition, frequency-dependent selection, adaptive dynamics, evolutionary branching, extinction, evolutionary bifurcation theory. 


\title{
About the Authors
}

\author{
S.A.H. Geritz \\ Department of Zoology \\ University of Maryland \\ College Park Maryland 20742 \\ USA \\ and \\ Institute of Evolutionary and Ecological Sciences \\ Kaiserstraat 63 \\ 2311 GP Leiden \\ The Netherlands \\ E. van der Meijden \\ Institute of Evolutionary and Ecological Sciences \\ Kaiserstraat 63 \\ 2311 GP Leiden \\ The Netherlands \\ J.A.J. Metz \\ Institute of Evolutionary and Ecological Sciences \\ Kaiserstraat 63 \\ 2311 GP Leiden \\ The Netherlands \\ and \\ Adaptive Dynamics Network \\ International Institute for Applied System Analysis| \\ 2361 Laxenburg, Austria
}

\section{Acknowledgments}

This work was supported by grants from the Netherlands Organization for Scientific Research (NWO) and from the University of Maryland at College Park. We thank Éva Kisdi, Peter Klinkhamer, Tom de Jong, Géza Meszéna, Frans Jacobs and Peter Abrams for many discussions on the topic of seed size evolution and evolutionary dynamics. 


\title{
Evolutionary Dynamics of Seed Size and Seedling Competitive Ability
}

\author{
S.A.H. Geritz \\ E. van der Meijden \\ J.A.J. Metz
}

\section{Introduction}

One of the most fundamental life-history trade-offs is between the size and the number of offspring. With a given amount of resources only a limited number of offspring of finite size can be produced. The more one particular offspring gets, the less will be available for the others, so that the number of offspring can be increased only at the cost of decreasing average offspring size. Offspring size, however, tends to be positively correlated with offspring survival or future offspring fecundity in such various organisms as, for example, crustaceans (Parish and Pitelka, 1962; Jones, 1978), birds (Perrins, 1965), amphibians (Arnold, 1983), human beings (Schluter, 1988), and plants (Stanton, 1984; Winn, 1988; Mogie et al., 1990; Bell et al., 1991; Cipollini and Styles, 1991; Houssard and Escarré, 1991; Jurado and Westoby, 1992; Osunkoya et al., 1994). Smith and Fretwell (1974) formulated a model for the optimal balance between the size and the number of offspring. In their model, the fitness, $W$, of a reproducing individual with a total amount of $R$ resources and offspring of size $m$, is given by

$$
W(m)=f(m) \frac{R}{m}
$$

where $f(m)$ is the expected reproductive yield per offspring of size $m$, and $R / m$ is the total number of offspring produced. Independently of what $f(m)$ exactly looks like, there is always a single (globally) optimal offspring size that maximizes fitness. The optimal size, $m_{\text {opt }}$, coincides with the point of contact where a straight line through the origin touches the graph of $f(m)$ (Figure 1a), and is independent of the amount of resources available. Thus, according to the Smith-Fretwell (1974) model, parental fitness is maximized by investing equally in all offspring, so that any variation in offspring size should be explained as a constraint on the parent's capability to produce offspring of uniform size rather than as an adaptation.

Different shapes of $f(m)$ correspond to different species or different environments. The Smith-Fretwell (1974) model thus could explain variation in offspring size between species and between different habitats of the same species. 

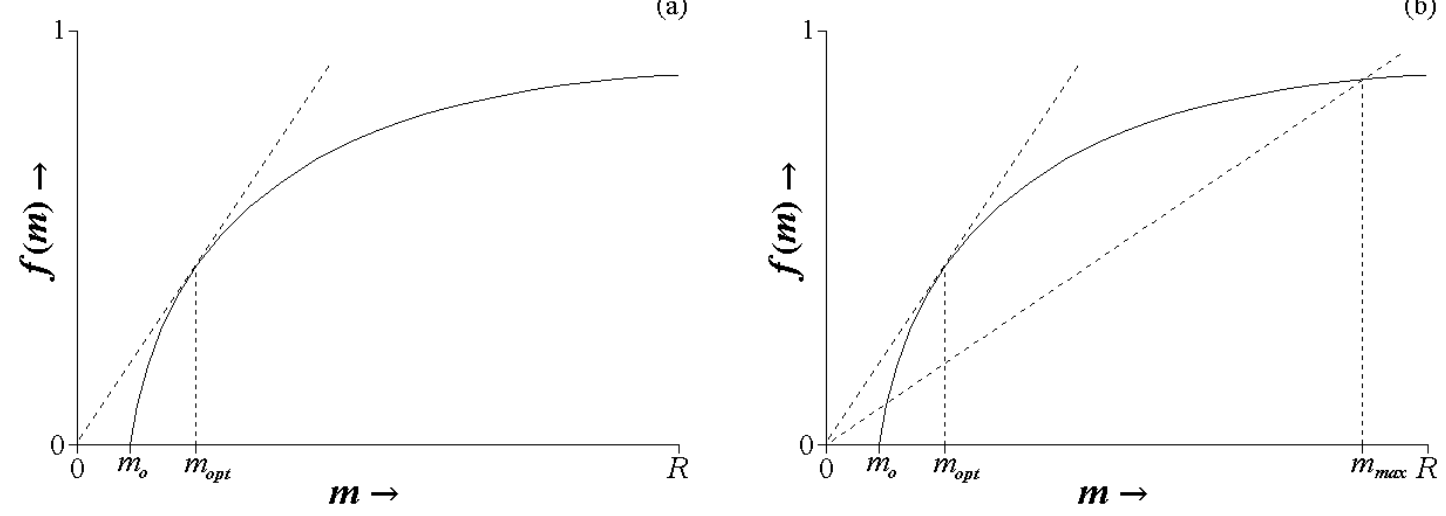

Figure 1. (a) Optimization of offspring size in the Smith-Fretwell (1974) model. The optimal offspring size, $m_{\text {opt }}$, coincides with the point of contact where the graph of $f(m)$ is touched by a straight line through the origin. (b) Evolutionarily stable seed size distribution in the model of Geritz (1995). The ESS includes all seed sizes between the Smith-Fretwell optimum, $m_{\mathrm{opt}}$, and $m_{\max }$, which is given the intersection of the graph of $f(x)$ with the main diagonal. The smallest seeds that still produce viable seedlings have size $m_{\mathrm{o}}$.

Within the context of seed size and seed number strategies in plants, however, the model has been criticized for its incapability to explain seed size variation within plant populations of the same species and within individual plants. Although seed size traditionally has been considered a remarkably constant characteristic relative to other features such as plant height or seed number (Harper et al., 1970), seed size variation among, as well as within individual plants of a single population is nevertheless common and widespread (Michaels et al., 1988; Westoby et al., 1992). To account for this variation as a result of natural selection (rather than as a constraint), various alternative models have been proposed that include such factors as temporal variation in offspring environment (Venable, 1985; McGinley et al., 1987), spatial variation in offspring environment (McGinley et al., 1987; Garciá-Dorado, 1990), variation in the availability of resources during the offspring-provisioning period (Lalonde, 1991), variation of the genetic quality of the offspring (Temme, 1986), multiple resources (McGinley and Charnov, 1987), generalized size-number trade-offs and parental resource status (Venable 1992), seedling competition (Geritz, 1995; Rees and Westoby, 1997), variation in pollination success (Sakai, 1995; Sakai and Sakai, 1995), or seed predation (Geritz 1997). In this paper we investigate how different degrees of competitive asymmetry due to differences in seed size can affect the evolution of seed size variation.

The constancy of seed size in the Smith-Fretwell (1974) model is a consequence of the assumption that for any given environment the reproductive yield per seed is a fixed function of seed size that simultaneously takes account of all biotic and abiotic factors affecting the survival and expected fecundity of the offspring. This, however, no longer holds when seed size influences the outcome of competition among the seedlings. In various species, seed size has been shown experimentally to affect the competitive ability of seedlings in favor of larger seeds (Black, 1958; Stanton, 1985; Wulff, 1986b). Presumably this is due to large seeds having more reserves and a larger embryo than small seeds (Fenner, 1983; Wulff, 1986a), thus giving seedlings from large seeds a head-start in competition. The expected reproductive yield per offspring then not 
(a)

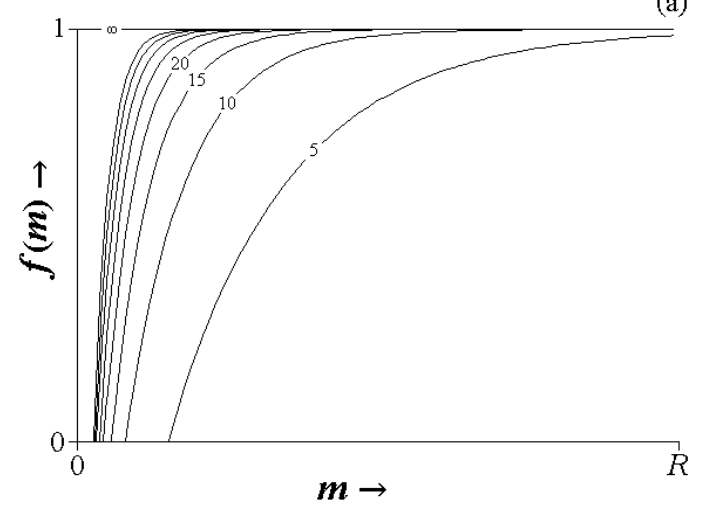

(b)

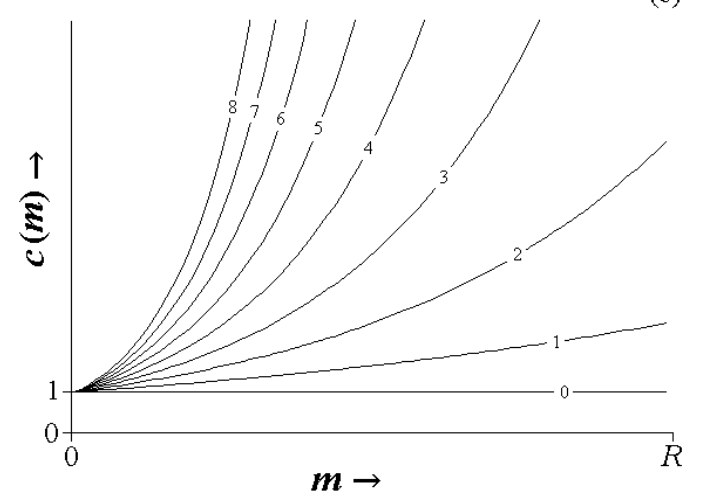

Figure 2. (a) Precompetitive seed and seedling survival, $f(m)$, for different values of $\beta R$ representing different types of environments. (b) Seedling competitive ability, $c(m)$, for different values of $\alpha R$ representing different levels of competitive asymmetry.

only depends on a plant's own seed size but on that of its competitors as well. Seedling competition, therefore, may lead to frequency-dependent selection, in which case simple, direct fitness maximization like in the model of Smith and Fretwell (1974) is no longer possible and a game-theoretical approach (Maynard Smith 1982) should be used instead.

To investigate the evolutionary consequences of seedling competition, we use an extension of the Smith-Fretwell (1974) model based on a competition model for annual plants without a seed bank as described by Geritz et al. (1988) and Geritz (1995). In the model seeds are dispersed randomly into a large number of discrete germination sites such that the number of seeds landing in any one site is Poisson-distributed. During dispersal, germination and early seedling growth, the offspring first undergo a period of frequency-independent selection due to predation, burial, desiccation and so forth. The survival probability for seeds of size $m$ during this period of frequency-independent selection is denoted by $f(m)$, and is assumed to be given by

$$
f(m)=\max \{0,1-2 \cdot \exp (-\beta m)\}
$$

(Figure 2a). Different values of $\beta$ correspond to different habitat types: high values of $\beta$ are typical for open, moist conditions that favor seedling establishment for both small and large seeds (Gross and Werner, 1982; Gross, 1984; Stanton, 1984; Wulff, 1986a), whereas low values of $\beta$ correspond to shaded conditions under perennial vegetation or litter where seedling establishment is low and where large seeds have a significant precompetitive advantage relative to smaller seeds (Grime and Jeffrey, 1965; Haskins and Gorz, 1975; Gross and Werner, 1982; Gross, 1984; Winn, 1985). In the limit for $\beta=$ $\infty$, all seeds survive and produce established plants, irrespectively of their size (Figure 2a).

As $f(m)$ takes account of all frequency-independent fitness aspects of seed size, it has essentially the same meaning as in the model of Smith-Fretwell (1974). Additional, non-selective precompetitive mortality due to seed landings outside of suitable germination sites, trampling or flooding, and so forth, independently of seed size, can be accounted for by a lower total amount of available resources per germination site. 
Seedlings that survive the initial phase of frequency-independent selection compete for the locally available resources within a germination site. The individual seedlings may have different expected future fecundities depending on the amount of resources obtained. The total amount of available resources per germination site is denoted by $R$. We assume that in a site with a total number of $k$ competitors with, respectively, seeds of sizes $m_{1}, \ldots, m_{k}$, the fraction of $R$ gained by an individual seedling with seed size $m_{i}(i=1, \ldots, k)$ is given by

$$
\frac{c\left(m_{i}\right)}{c\left(m_{1}\right)+\cdots+c\left(m_{k}\right)}
$$

where $c(m)$ is a measure of the competitive ability of a seedling with seed size $m$. Alternatively, Equation (3) can be interpreted as the probability of establishment when there is room for only one established plant per germination site, so that competition affects survival rather than fecundity. The two interpretations are mathematically equivalent, however, and give exactly the same demographic and evolutionary outcomes. Models using Equation (3) are called lottery competition models or safe-site models (for other examples of lottery competition and safe-site models, see, e.g., Chesson and Warner, 1981; Fagerström and Ågren, 1979; Ågren and Fagerström, 1984; Geritz et al., 1988; Geritz, 1995).

For various species it has been shown empirically that an increase in seed size gives a disproportionate large increase in an individual's total reproductive output (Stanton, 1985; Wulff, 1986b). Presumably this is due to differences in relative growth rates during competition when large seedlings have better access to light and nutrients than small seedlings as a consequence of shading and a more extended root system (Ford, 1975; Weiner, 1990). Small initial differences in seedling size resulting from differences in seed size thus tend to become enlarged during competition, giving a disproportionately competitive advantage to large seeds. In other words, seedling competitive ability is likely to be a convex and increasing function of seed size. We here assume that $c(m)$ is given by

$$
c(m)=\exp (\alpha m)
$$

(Figure 2b), where $\alpha$ is equal to the proportional increase in competitive ability due to an increase in seed size. The parameter $\alpha$ determines the level of competitive asymmetry: if $\alpha$ is zero, seed size has no effect on the competitive ability of seedlings, and all seedlings in a given germination site get the same amount of resources irrespective of their seed size (i.e., symmetric competition). Differences in fitness between plants with different seed sizes are then due only to differences in precompetitive seed and seedling survival, $f(m)$, so that we effectively recover the Smith-Fretwell (1974) model. Thus, with symmetric competition, we always find a single optimal seed size excluding all other seed sizes (cf. Figure 1a).

As $\alpha$ increases, however, the competitive advantage of large seeds increases. In the limit for $\alpha$ approaching infinity, only the single seedling from the largest seed present in a given germination site obtains resources, while all other seedlings in the same site get none at all and thus fail to reproduce (i.e., extremely asymmetric competition). With extremely asymmetric competition there is no longer a single, 
optimal seed size, because a population with a single seed size can always be invaded by plants with slightly larger seeds or by plants with sufficiently smaller but (due to the size-number trade-off) more numerous seeds (Geritz et al., 1988). Instead, there is an evolutionarily stable seed polymorphism with a continuum seed sizes (Figure 1b). Plants with small seeds survive because they are better colonizers (due to their larger seed number) which enables them to exploit sites that by chance remain unoccupied by the larger (but less numerous) seeds of their competitors (Geritz, 1995).

In this paper we investigate the evolution of seed size and seedling competitive ability for intermediate levels of competitive asymmetry $(\alpha)$, different types of precompetitive environment $(\beta)$, and different amounts of resources available per germination size $(R)$. We explore how the two extreme outcomes of the model (i.e., a single optimal seed size with symmetric competition on the one hand, and a continuum of coexisting seed sizes with extremely asymmetric competition on the other) are connected to one another via intermediate levels of competitive asymmetry. At intermediate levels of competitive asymmetry the model is very hard to analyze algebraically, and we therefore resort to numerical analyses instead. To this end we assume that each individual plant produces seeds of one size only, and that the offspring always have the same seed size as the mother plant. In the Discussion we shall argue how the results can be interpreted in the context of a more realistic reproductive biology. A population with various coexisting plant types, each with its own particular seed size, is assumed to be always a protected polymorphism in the sense that each plant type present is protected against extinction by an annual per capita growth rate that is larger than one when rare. Moreover, we assume that phenotypic mutations in seed size occur only very infrequently, and only one at a time, so that the resident population has reached its demographic equilibrium before the next mutant comes along. We also assume that the resident population is large enough that we can ignore demographic stochasticity. Finally, we assume that the effect of a single mutation on seed size is small but finite so that evolution proceeds by small but discrete individual steps. We analyze the model within the context of a theoretical framework for modeling evolutionary dynamics developed by Metz et al. (1996) and Geritz et al. (1997a,b).

\section{Evolutionarily Singular Strategies}

\section{Resident population dynamics and mutant's fitness}

Consider a resident population with $n$ different plant types, each with its own seed size denoted by $m_{i}(i=1, \ldots, n)$, and let $N_{i, t}$ denote the density in year $t$ of plants with seed size $m_{i}$ expressed as the average number of established plants per germination site. The fraction of resources obtained per seedling with seed size $m_{i}$ in a germination site together with $k_{1}, \ldots, k_{n}$ seedlings with, respectively, seeds of sizes $m_{1}, \ldots, m_{n}$ is given by

$$
\frac{c\left(m_{i}\right)}{c\left(m_{i}\right)+k_{1} c\left(m_{1}\right)+\cdots+k_{n} c\left(m_{n}\right)}
$$

(cf. Equation 3). Modeling a trade-off between seed size and seed number in the same way as in the model of Smith and Fretwell (1974), and taking into account that only a fraction $f\left(m_{i}\right)$ of all seeds of size $m_{i}$ survive the precompetitive phase and eventually 
become established plants, the number of established offspring produced by a plant with seed size $m_{i}$ in a germination site together with $k_{1}, \ldots, k_{n}$ other plants with seeds of sizes $m_{1}, \ldots, m_{n}$ is

$$
f\left(m_{i}\right) \frac{R}{m_{i}}\left(\frac{c\left(m_{i}\right)}{c\left(m_{i}\right)+k_{1} c\left(m_{1}\right)+\cdots+k_{n} c\left(m_{n}\right)}\right) .
$$

Assuming Poisson-distributed seed numbers per germination site, the numbers of competing seedlings per germination site, $k_{1}, \ldots, k_{n}$, are also Poisson-distributed with expectations $N_{1, t}, \ldots, N_{n, t}$, so that the expected number of established offspring per plant with seed size $m_{i}$ is given by

$$
f\left(m_{i}\right) \frac{R}{m_{i}} \cdot\left(\sum_{k_{1}=0}^{\infty} \cdots \sum_{k_{n}=0}^{\infty} \frac{c\left(m_{i}\right)}{c\left(m_{i}\right)+k_{1} c\left(m_{1}\right)+\cdots+k_{n} c\left(m_{n}\right)} \frac{\left(N_{1, t}\right)^{k_{1}} \cdots\left(N_{n, t}\right)^{k_{n}}}{k_{1} ! \cdots k_{n} !} e^{-N_{1, t}-\cdots-N_{n, t}}\right) .
$$

By rescaling seed size as the fraction of the available resources per germination site (i.e., using $m_{i} / R$ instead of $m_{i}$ as a relative measure of seed size) it can be seen from Equations (2), (4), and (6) that the outcome of the model will depend only on the two products $\alpha R$ and $\beta R$ instead of each parameter individually.

At demographic equilibrium the expected per capita number of established offspring (Equation 6) is equal to one for all plant types present in the population. In a monomorphic resident population with only seeds of size $m$, there is a single, positive and asymptotically stable equilibrium density whenever $f(m) R / m>1$ (Geritz et al., 1988). The equilibrium depends on the resident's seed size and hence will be denoted by $N(m)$. If $f(m) R / m \leq 1$, the per capita number of established offspring is too low to maintain a positive population density.

Next, consider a mutant plant with seed size $m^{\prime}$ in a monomorphic resident population at its equilibrium with seed size $m$. During the early phase of invasion when the mutant is still rare, the probability of two or more mutant seeds landing in the same germination site is negligible. Mutant seedlings, therefore, initially compete only with resident seedlings and not among themselves. For the mutant's per capita number of established offspring, that is, its fitness, denoted by $W_{m}\left(m^{\prime}\right)$, we thus find

$$
W_{m}\left(m^{\prime}\right)=f\left(m^{\prime}\right) \frac{R}{m^{\prime}} \cdot\left(\sum_{k=0}^{\infty} \frac{c\left(m^{\prime}\right)}{c\left(m^{\prime}\right)+k \cdot c(m)} \frac{(N(m))^{k}}{k !} e^{-N(m)}\right)
$$

(cf. Equation 6). Notice that $W_{m}\left(m^{\prime}\right)$ is equal to the mutant's fitness, $f\left(m^{\prime}\right) R / m^{\prime}$, as in the Smith-Fretwell (1974) model (cf. Equation 1) times a correction factor that takes account of the effects of competition. Other authors also use the logarithmic growth rate, that is, $\ln \left(W_{m}\left(m^{\prime}\right)\right)$, as a measure of the mutant's fitness (see, e.g., Metz et al., 1996; Geritz et al., 1997a,b; Meszéna et al., 1997).

If $W_{m}\left(m^{\prime}\right)<1$, the mutant dies out. If $W_{m}\left(m^{\prime}\right)>1$, the mutant may spread (but need not always do so, because of the possibility of random extinction due to drift when there are still only very few copies of the mutant around). If the mutant spreads and in addition we have $W_{m^{\prime}}(m)<1$ (so that the resident cannot recover once rare itself), then the mutant will eventually replace the resident and take over the population. We shall 
(a)

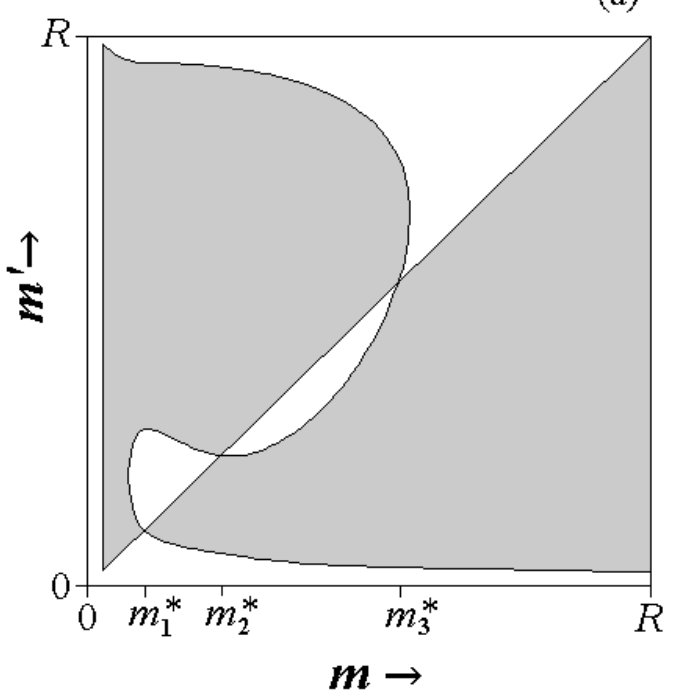

(b)

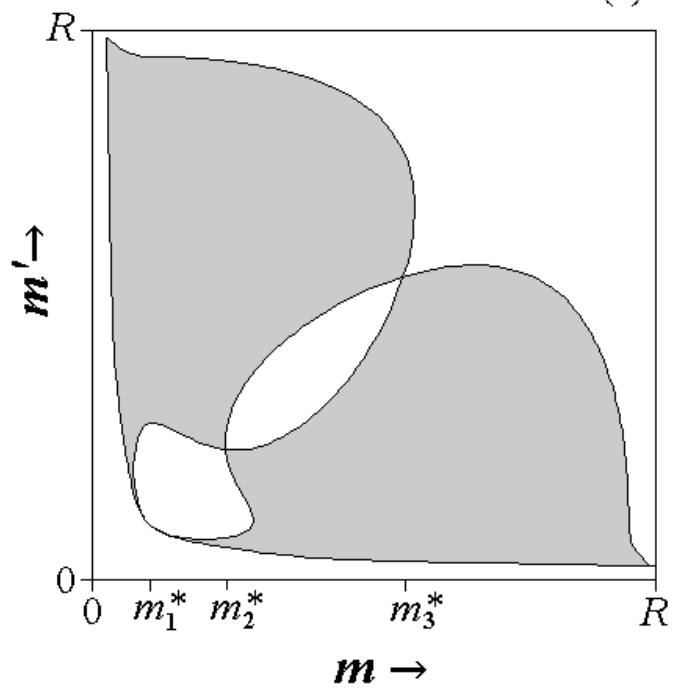

Figure 3. (a) Pairwise invasibility plot for $\alpha R=6$ and $\beta R=25$. Shaded regions indicate where $W_{m}\left(m^{\prime}\right)>1$. (b) Area of coexistence for the same parameter values. The shaded regions indicate combinations of $m$ and $m^{\prime}$ that can mutually invade, and hence can coexists as a protected dimorphism.

refer to this as a substitution. If, however, the mutant spreads but in addition $W_{m^{\prime}}(m)>1$ (so that the resident is protected against extinction and cannot be replaced), then the population necessarily becomes dimorphic with both the resident and the mutant living together. We shall refer to this as mutual invasibility of the two seed sizes.

\section{Evolutionarily stable singular strategies}

Figure 3a shows a so-called pairwise invasibility plot for $\alpha R=6, \beta R=25$. In this graph we indicate for what combinations of resident and mutant seed sizes $W_{m}\left(m^{\prime}\right)$ is larger or smaller than one. (For other examples of pairwise invasibility plots, see, e.g., Van Tienderen and De Jong, 1986; Metz et al., 1992; Kisdi and Meszéna, 1993, 1995.) To see what mutants can spread in a given resident population we look along a vertical line through the point on the $m$-axis representing the resident's seed size. The parts of this line inside a region for which $W_{m}\left(m^{\prime}\right)>1$ (shaded regions) correspond to potentially invading mutants, and the parts of the line inside a region for which $W_{m}\left(m^{\prime}\right)<1$ (unshaded regions) correspond to mutants that cannot invade. With small evolutionary steps we need only to consider combinations of seed sizes inside a narrow region along the main diagonal. As residents are selectively neutral among themselves, on the main diagonal (where the mutant and the resident have the same seed size) $W_{m}\left(m^{\prime}\right)$ is necessarily equal to one. Intersections of the diagonal with other lines on which $W_{m}\left(m^{\prime}\right)$ $=1$ correspond to so-called evolutionarily singular strategies (Metz et al., 1996; Geritz et al., 1997a,b). On the diagonal of the pairwise invasibility plot at a singular strategy, the mutant's fitness as a function of its own seed size attains either a local maximum or a local minimum. Algebraically, therefore, a singular strategy is characterized by

$$
\left[\frac{\partial W_{m}\left(m^{\prime}\right)}{\partial m^{\prime}}\right]_{m^{\prime}=m}=0
$$


In Figure 3a there are three evolutionarily singular strategies, namely, $m_{1}{ }^{*}, m_{2}{ }^{*}$, and $m_{3}{ }^{*}$. Looking along a vertical line through $m_{1}{ }^{*}$, it can be seen that no nearby mutants can invade a resident population in which all plants have seed size $m_{1}{ }^{*}$. In other words, $m_{1} *$ is a (locally) evolutionarily stable strategy, or ESS (Maynard Smith, 1982). On the diagonal of the pairwise invasibility plot at an evolutionarily stable singular strategy, the mutant's fitness as a function of its own seed size has a (local) maximum. An evolutionarily stable singular strategy is therefore algebraically characterized by

$$
\left[\frac{\partial^{2} W_{m}\left(m^{\prime}\right)}{\partial m^{\prime 2}}\right]_{m^{\prime}=m}<0
$$

(Maynard Smith, 1982). Notice that $m_{1} *$ is not a global ESS, because mutants further away (i.e., between $m^{\prime}=0.29 R$ and $m^{\prime}=0.95 R$ ) in fact could invade if only they happened to appear. Assuming sufficiently small evolutionary steps, however, these seed sizes cannot be generated by a single mutation of $m_{1}{ }^{*}$, and can therefore be ignored. Neither $m_{2}{ }^{*}$ nor $m_{3}{ }^{*}$ is (locally) evolutionarily stable, because both can be invaded by any (nearby) mutant (Figure 3a).

\section{Convergence stable singular strategies}

Figure $3 \mathrm{~b}$ shows what combinations of resident and mutant seed sizes can coexist as a protected dimorphism (shaded regions). The figure was obtained by first taking the mirror image of Figure 3a along its main diagonal (which gives all combinations of seed sizes for which $W_{m}(m)>1$ ), and putting it on top of the original. The overlapping parts of the shaded regions in the mirror image and in its original correspond to seed sizes for which both $W_{m}\left(m^{\prime}\right)>1$ and $W_{m^{\prime}}(m)>1$ at the same time and hence can coexist as a protected dimorphism. The set of possible protected dimorphisms (Figure 3b) we shall refer to as the area of coexistence. The area of coexistence reaches the diagonal only at the singular strategies $m_{1}{ }^{*}, m_{2}{ }^{*}$, and $m_{3}{ }^{*}$ (Figure $3 \mathrm{~b}$ ). Mutual invasibility and hence coexistence of two almost identical seed sizes can therefore only occur when the population is very close to a singular strategy and nowhere else. In other words, with small evolutionary steps (in which case mutants are always very similar to their resident progenitor) any mutant that can spread in a given resident population will always replace the resident, that is, as long as the population stays away from the singular strategies.

Starting with a resident population with a seed size smaller than $m_{1}{ }^{*}$, it can be seen from Figure $3 a$ that mutants with slightly larger seeds can invade (and subsequently replace the resident), whereas mutants with smaller seeds cannot. Similarly, a resident population on the other side of $m_{1}{ }^{*}$ can be invaded (and replaced) only by mutants with smaller seeds. In other words, with small evolutionary steps, $m_{1}{ }^{*}$ is an evolutionary attractor in the sense that an initially monomorphic population will gradually evolve towards $m_{1}{ }^{*}$. Evolutionary attractors in the above sense are called convergence stable (Eshel, 1983; Christiansen, 1991). At a convergence stable singular strategy, the slope of the mutant's fitness as a function of its own strategy, that is, the local fitness gradient,

$$
\left[\frac{\partial W_{m}\left(m^{\prime}\right)}{\partial m^{\prime}}\right]_{m^{\prime}=m}
$$


changes its sign from positive on the left side of the attractor to negative on the other side (Figure 3a), and therefore is a locally decreasing function of seed size. A convergence stable singular strategy is thus algebraically characterized by

$$
\frac{d}{d m}\left\{\left[\frac{\partial W_{m}\left(m^{\prime}\right)}{\partial m^{\prime}}\right]_{m^{\prime}=m}\right\}=\left[\frac{\partial^{2} W_{m}\left(m^{\prime}\right)}{\partial m \partial m^{\prime}}\right]_{m^{\prime}=m}+\left[\frac{\partial^{2} W_{m}\left(m^{\prime}\right)}{\partial m^{\prime 2}}\right]_{m^{\prime}=m}<0
$$

(Eshel, 1983). Equivalent conditions have been used by Metz et al. (1996) and Geritz et al. (1997a,b). From Figure $3 \mathrm{a}$ it can also be seen that $m_{2}{ }^{*}$ lacks convergence stability (and thus is an evolutionary repeller from which an initially monomorphic resident population tends to evolve away), whereas $m_{3}{ }^{*}$, again, is convergence stable. Once a population has come sufficiently close to a convergence stable singular strategy, sooner or later a mutant may come along that no longer can replace the resident (Figure 3b), so that the population becomes dimorphic. The significance of dimorphisms near a singular strategy is discussed below.

\section{Evolutionary branching}

Evolutionary stability and convergence stability are fully independent properties that can occur in any combination (Eshel and Motro, 1981; Eshel, 1983; Taylor, 1989; Christiansen, 1991; Abrams et al., 1993; Eshel et al., 1997). The evolutionary significance of protected dimorphisms arising nearby a singular strategy depends on the particular combination of the two types of stability. If a singular strategy is both convergence stable and evolutionarily stable, like in the case of $m_{1}{ }^{*}$ in Figure 3a, only mutants with a seed size in between the two resident seed sizes can invade, so that with each successive invasion the dimorphism is replaced by either a monomorphism or a dimorphism that is even closer to the singular strategy than before (Metz et al., 1996; Geritz et al., 1997a,b). In the long run, therefore, any nearby dimorphism effectively disappears again as the population continues to converge to the singular strategy through a series of alternating monomorphic and dimorphic population states. A singular strategy that is both evolutionarily stable and convergence stable is called a continuously stable strategy, or CSS (Eshel and Motro, 1981; Eshel, 1983), and represents a possible final, monomorphic stop of the evolutionary process. In the case of $m_{2}{ }^{*}$, however, which is an evolutionary repeller, the population most probably will evolve away before it has a chance of becoming dimorphic. Its main significance is that it separates the domains of attraction of $m_{1}^{*}$ and $m_{3}^{*}$.

The case of $m_{3}^{*}$, which is convergence stable but not evolutionarily stable, stands out in particular. For a monomorphic population it acts as an evolutionary attractor. At the singular strategy, however, the mutant's fitness as a function of its own strategy has a local minimum. As soon as the population reaches the singular strategy and becomes dimorphic, it undergoes disruptive selection, forcing the two resident seed sizes to evolve further and further apart with each successive invasion (Metz et al., 1996; Geritz et al., 1997a,b; Eshel et al. 1997). This process of phenotypic divergence in an initially monomorphic resident population we call evolutionary branching, and a singular strategy that is convergence stable but at the same time lacks evolutionary stability we call an evolutionary branching point. 
(a)

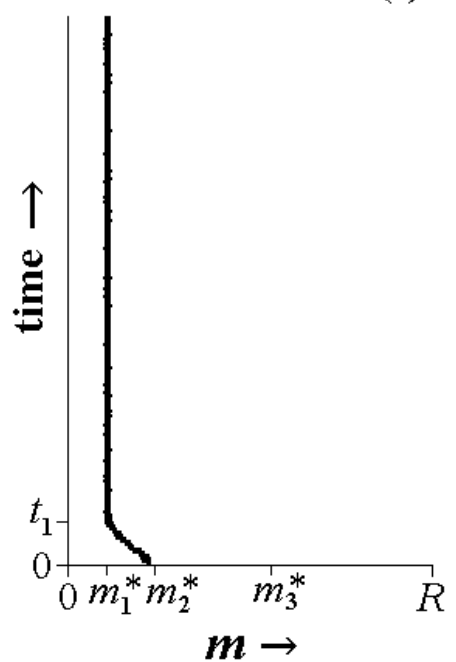

(b)

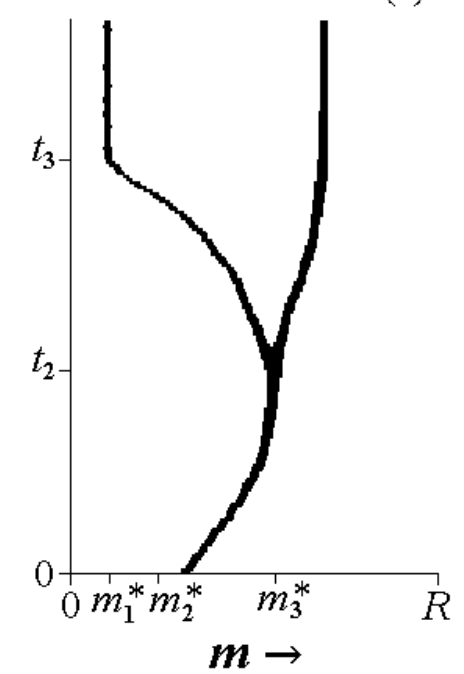

Figure 4. Two simulated evolutionary trees with different initial seed sizes for $\alpha R=6$ and $\beta R=25$. (a) The population starting left of the repeller $m_{3} *$ reaches the evolutionarily stable seed size $m_{1} *$ at time $t_{1}$. (b) The population that starts on the right side of $m_{2}{ }^{*}$ evolves to the branching point $m_{3}{ }^{*}$ and undergoes disruptive selection at time $t_{2}$. The population reaches a dimorphic evolutionarily stable state at time $t_{3}$.

Figure 4 shows the results of numerical simulations of the evolutionary process using the per capita number of established offspring given in Equation (6) to calculate the change in plant density from one generation to the next in a population with an arbitrary number of different plant types. Starting with a monomorphic population at the beginning of the simulation (i.e., $n=1$ ), new mutants are generated with a low probability per offspring produced by a small but random mutation from types already present. With each new mutation an extra equation for the mutant's population density is added to the system (i.e., $n$ is increased by one). New mutants are introduced initially at a very low frequency, but may increase or decrease during subsequent generations. When the frequency of a given type falls below a certain predefined threshold, it is considered to have gone extinct and is removed from the population.

The simulations confirm the predictions of the theory: depending on the initial seed size, the population either evolves towards $m_{1} *$ (Figure $4 \mathrm{a}$ ), or evolves first towards $m_{3}{ }^{*}$ where it undergoes evolutionary branching and eventually becomes distinctively dimorphic (Figure 4b). Neither the precise density at which new mutants are introduced or are eliminated from the population, nor the exact frequency and the magnitude of mutations qualitatively affect the outcomes of the simulations. Moreover, in the simulations new mutants often come along before the resident population has reached its equilibrium and before inferior mutants have been eliminated, which demonstrates that the assumptions of separate time scales for selection and mutation, and of a polymorphism always being a protected polymorphism, are not very critical. 


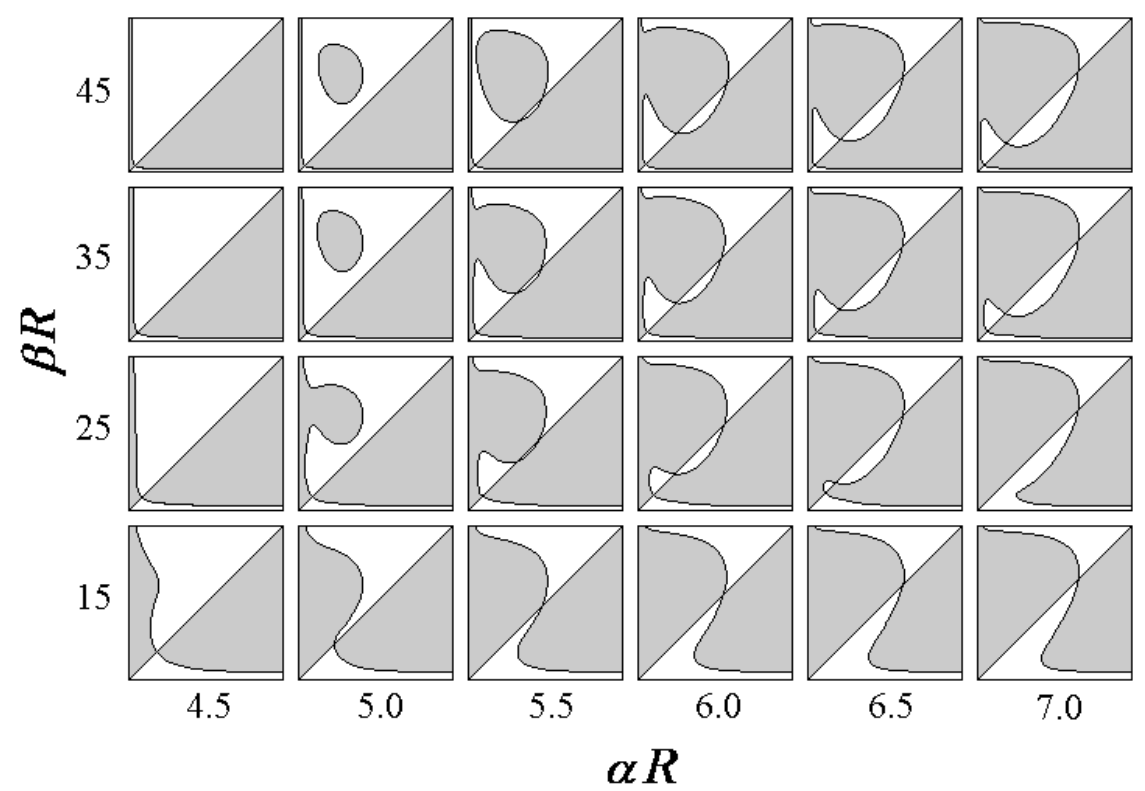

Figure 5. Pairwise invasibility plots for different values of $\alpha R$ and $\beta R$. In each plot the scale of the axis runs from zero to $R$.

\section{Changes in number and stability properties of evolutionary singular strategies}

The number and the stability properties of the singular strategies depend on the values of $\alpha R$ and $\beta R$. Figure 5 shows pairwise invasibility plots for various combinations of different parameter values. We first consider the row with $\beta R=25$. At low values of $\alpha R$, there is only a single, convergence stable and globally evolutionarily stable singular strategy (Figure $5 ; \alpha R=4.5, \beta R=25$ ). At higher values of $\alpha R$, the singular strategy loses its global evolutionary stability and becomes a local ESS $(\alpha R=5.0, \beta R=25)$. As $\alpha R$ increases further, two new singular strategies (i.e., a repeller and a branching point) appear when the curved line defined by $W_{m}\left(m^{\prime}\right)=1$ first touches and then intersects the main diagonal at two additional points $(\alpha R=5.5,6.0$ and 6.5, $\beta R=25)$. In a similar but reversed process at still higher values of $\alpha R$, the lower two intersection points with the main diagonal move closer together and finally merge and annihilate one another, so that in the end only the branching point remains $(\alpha R=7.0, \beta R=25)$. Just before the two singularities meet and disappear, however, the ESS first loses its evolutionary stability and becomes a branching point (Figure 6).

At higher values of $\beta R$, increasing $\alpha R$ may lead to the simultaneous appearance of the repeller and the branching point before the ESS loses its global stability (e.g., Figure 5; $\alpha R=5.5, \beta R=45$ ). The ESS then is globally evolutionarily stable but only locally convergence stable, which is possible because of the independence of evolutionary stability and convergence stability. At lower values of $\beta R$, the ESS may lose its global and local evolutionary stability without the appearance of additional singular strategies (e.g., Figure 5; $\alpha R=5.0, \beta R=15$ and $\alpha R=5.5, \beta R=15$ ).

Figure $7 \mathrm{a}$ shows how the number and stability properties of the singular strategies change as $\alpha R$ and $\beta R$ vary continuously. The figure was produced using 
(a)

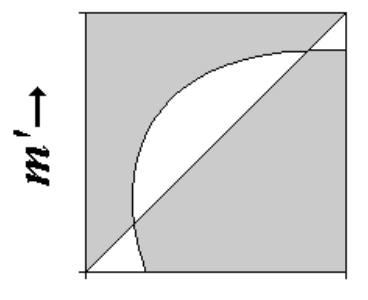

$m \rightarrow$

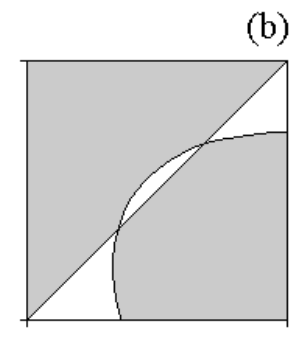

$m \rightarrow$

(b)

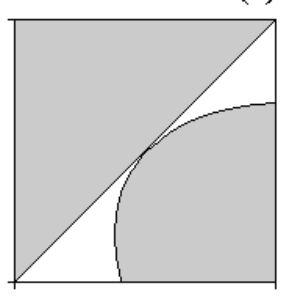

$m \rightarrow$ (c)

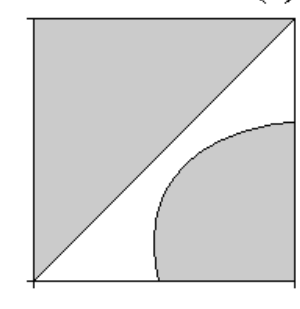

$m \rightarrow$

(d)

Figure 6. Detail of pairwise invasibility plots for (a) $\alpha R=6.754$, (b) $\alpha R=6.763$, (c) $\alpha R=6.766$ and (d) $\alpha R=6.769$ and fixed $\beta R=25$. The ESS in (a) must first lose its evolutionary stability in (b) before it collides with the repeller in (c), resulting in their mutual annihilation in (d). (Scales of the axis between $0.13 R$ to $0.15 R$.)

Equations (8), (9) and (11) to numerically identify and characterize singular strategies in terms of their stability properties. For parameter values inside the hatched region the precompetitive seed and seedling survival is too low for maintaining a positive population density, that is, $f(m) R / m$ is always smaller than one. The seed size that maximizes fitness in the Smith-Fretwell (1974) model is then optimal in the sense that it minimizes the rate of population decline. For parameter values above the hatched region, but left of the dotted line, there is a single, convergence stable and globally evolutionarily stable singular strategy. On the other side of the dotted line the ESS has lost its global character and has become only a local ESS. The thin solid line indicates where the ESS loses its evolutionary stability altogether and turns into a branching point. Only at point "P" where the dotted line and the thin solid line touch one another, the ESS loses both its global and local evolutionary stability at the same time and turns directly into a branching point.

Inside the wedge-shaped domain formed by the thick solid lines there are three singular strategies. The left edge of the wedge indicates where the repeller and the branching point first appear when in the pairwise invasibility plot the curve on which $W_{m}\left(m^{\prime}\right)$ is equal to one touches and then intersects the main diagonal at two additional points. The right edge indicates where the repeller and the former ESS merge and disappear together. In classical bifurcation theory, the mutual annihilation of two singularities or the simultaneous appearance of two singularities as a consequence of changing model parameters is called a fold bifurcation. The tip of the wedge-shaped domain where the two fold bifurcations meet and cancel one another is a so-called cuspbifurcation point (see, e.g., Kuznetsov, 1995). The significance of point "Q" is discussed later.

Figure $7 \mathrm{~b}$ gives a horizontal cross-section through the wedge-shaped domain in Figure $7 \mathrm{a}$ for $\beta R=35$, showing the actual values of the singular strategies as functions of $\alpha R$. The thick solid line indicates ESSes, the thin solid line branching points, and the thin dashed line evolutionary repellers for different values of $\alpha R$. The change from a global ESS into a local ESS is indicated by point "S". The change from a local ESS into a branching point occurs just before the lower part of the curve bends up and connects to the repeller, but the resolution of the figure is too low to show this clearly. The two folds lie exactly in between the left and right edge of the wedge-shaped domain in 

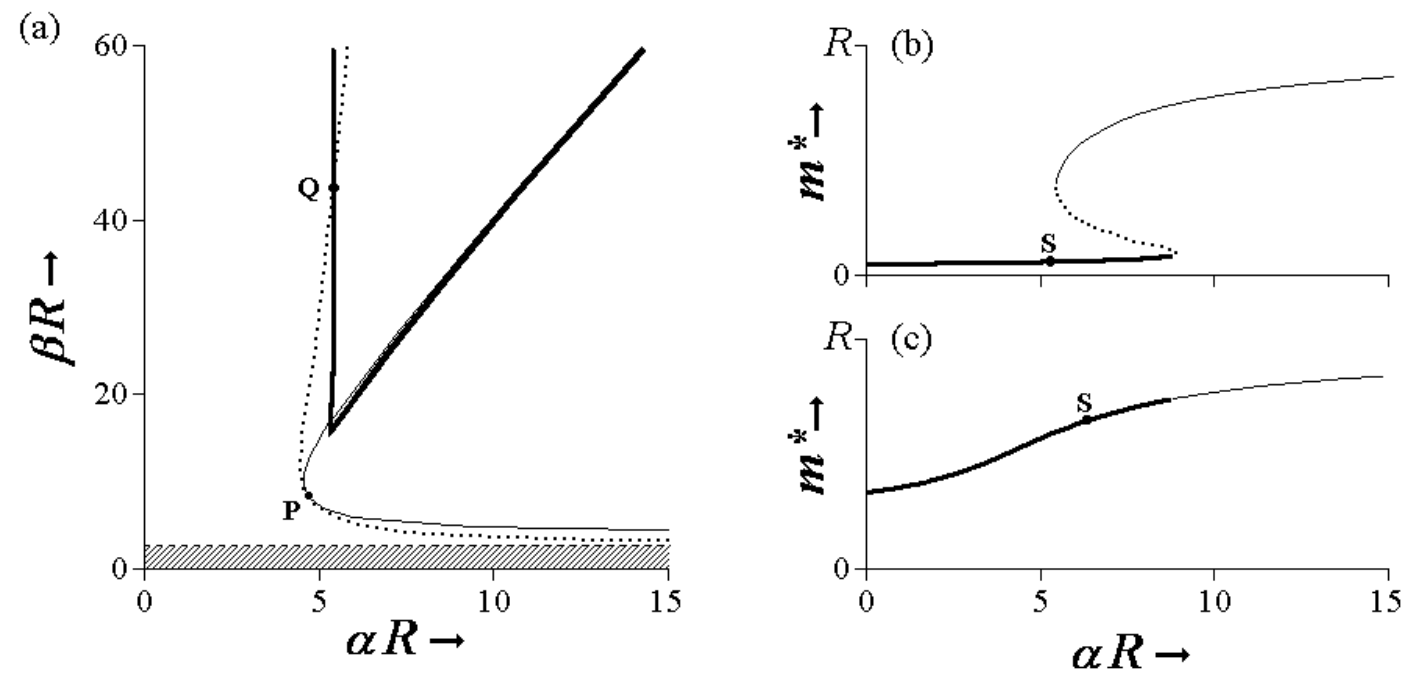

Figure 7. (a) Number and stability properties of the singular strategies as a function of $\alpha R$ and $\beta R$. (b) The actual values of the singular strategies for fixed $\beta R=35$, and (c) $\beta R=5$. (See text for further explanation.)

Figure 7a. As $\beta R$ decreases, the sides of the double fold becomes narrower, until at the cusp point only a vertical inflection point remains. Figure 7c is a cross-section at $\beta R=5$ illustrating that for values of $\beta R$ below the wedge-shaped domain in Figure 7 a the fold has completely disappeared and stretched out into a smooth, continuous line.

\section{Evolutionarily Singular Coalitions}

\section{Resident population dynamics and mutant's fitness}

To see what happens after evolutionary branching when the population has become distinctively dimorphic, we consider a resident population with two plant types with seed sizes $m_{1}$ and $m_{2}$. The full dynamics of the population follows from Equation (6) with $n=2$. Coexistence of two plant types is possible only if $m_{1}$ and $m_{2}$ are inside the area of coexistence (cf. Figure 3b). Geritz et al. (1988) showed that if coexistence is possible, then there is a single, asymptotically stable population dynamical equilibrium with both types present at positive densities. The average number of established plants per germination site at the equilibrium depends on $m_{1}$ and $m_{2}$, and will be denoted by respectively $N_{1}\left(m_{1}, m_{2}\right)$ and $N_{2}\left(m_{1}, m_{2}\right)$. At the boundary of the area of coexistence where $W_{m 1}\left(m_{2}\right)=1$ or $W_{m 2}\left(m_{1}\right)=1$, the equilibrium density of respectively $m_{2}$ and $m_{1}$ becomes zero.

The fitness, $W_{m 1, m 2}\left(m^{\prime}\right)$, of an initially rare mutant with seed size $m^{\prime}$ in a resident population with seed sizes $m_{1}$ and $m_{2}$ at its equilibrium is derived in a similar way as in the case of a monomorphic population, and is found to be

$$
\begin{aligned}
& W_{m_{1}, m_{2}}\left(m^{\prime}\right)= \\
& f\left(m^{\prime}\right) \frac{R}{m^{\prime}} \cdot\left(\sum_{k_{1}, k_{2}=0}^{\infty} \frac{c\left(m^{\prime}\right)}{c\left(m^{\prime}\right)+k_{1} c\left(m_{1}\right)+k_{2} c\left(m_{2}\right)} \frac{N_{1}\left(m_{1}, m_{2}\right)^{k_{1}} N_{2}\left(m_{1}, m_{2}\right)^{k_{2}}}{k_{1} ! k_{2} !} e^{-N_{1}\left(m_{1}, m_{2}\right)-N_{2}\left(m_{1}, m_{2}\right)}\right)
\end{aligned}
$$


(cf. Equation 7). The mutant can invade if $W_{m 1, m 2}\left(m^{\prime}\right)>1$, and dies out if $W_{m 1, m 2}\left(m^{\prime}\right)<1$. We shall without loss of generality assume that it was $m_{1}$ that mutated. After the mutant has invaded, the population dynamics can in essence follow four possible patterns. The most common pattern (i) is that the mutant just ousts the resident to which it is most similar, that is, its progenitor $m_{1}$. We shall call this a simple substitution. Apart from substitutions there are three other patterns that are relatively more rare, but that do occur regularly in the course of the evolutionary trajectories, and are moreover essential in determining the shape of those trajectories. In pattern (ii) all three types coexist until the occurrence of the next successful mutant, which for $m^{\prime}$ close to $m_{1}$ is only possible near the so-called evolutionary isocline for $m_{1}$ (to be discussed in the next paragraph). Pattern (iii) occurs only when the population is near the boundary of the area of coexistence where the frequency of $m_{2}$ becomes zero and the combination of $m^{\prime}$ and $m_{2}$ falls just outside the area of coexistence. In this case the mutant replaces both former resident types, so that the population becomes monomorphic again. We shall refer to this as an extinction event. Finally, in pattern (iv) $m_{2}$ dies out while $m^{\prime}$ and $m_{1}$ remain, which is only possible at the confluence of the conditions that we gave for patterns (ii) and (iii). Although trajectories containing pattern (iv) have but a negligible small probability to occur, and have moreover never been observed for the present model, the possibility cannot a priori be excluded.

\section{Isoclines and evolutionarily singular coalitions}

Since by assumption mutations occur only one at a time, a single evolutionary step inside the area of coexistence is always either horizontal or vertical depending on which particular resident produces the next successfully invading mutant first. With small evolutionary steps, the direction of a possible change in $m_{i}(i=1,2)$ is given by the sign of the local fitness gradient,

$$
\left[\frac{\partial W_{m_{1}, m_{2}}\left(m^{\prime}\right)}{\partial m^{\prime}}\right]_{m^{\prime}=m_{i}}
$$

(cf. Equation 10). The fitness gradients of $m_{1}$ and $m_{2}$ together define the so-called invasion cone, that is, the set of admissible evolutionary change given the present state of the resident population (Matessi and Di Pasquale, 1996). Figure 8 shows the area of coexistence for $\alpha R=6$ and $\beta R=25$ with the invasion cones indicated by arrows. Lines inside the area of coexistence on which the local fitness gradient for $m_{i}$ is zero we refer to as the evolutionary isocline for $m_{i}$, or simply the $m_{i}$-isocline. In a resident population on the $m_{i}$-isocline, the mutant's fitness as a function of its own seed size has either a local maximum for $m^{\prime}=m_{i}$, that is, if

$$
\left[\frac{\partial^{2} W_{m_{1}, m_{2}}\left(m^{\prime}\right)}{\partial m^{\prime 2}}\right]_{m^{\prime}=m_{i}}<0,
$$

or a local minimum if the reverse is true. Parts of the isoclines corresponding to local fitness maxima are indicated by thick solid lines, whereas parts corresponding to fitness minima by thin lines (Figure 8). 


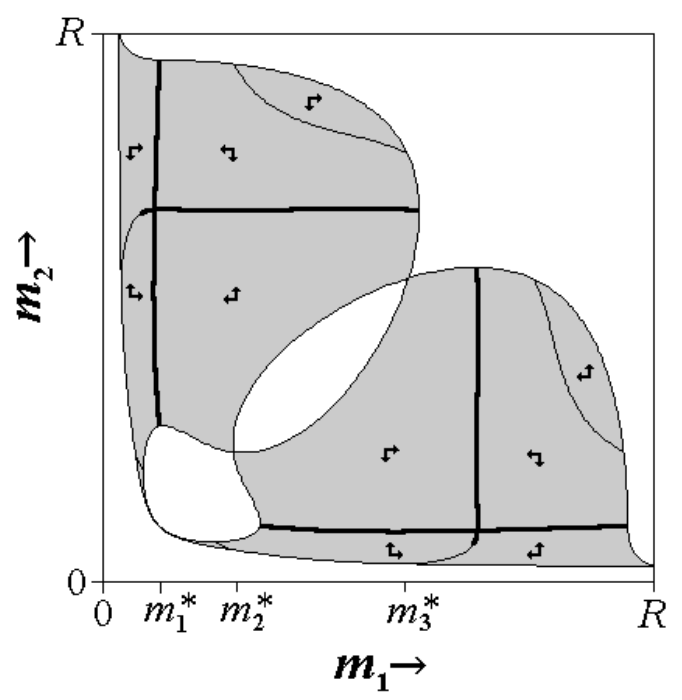

Figure 8. Area of coexistence with isoclines and invasion cones for $\alpha \mathrm{R}=6$ and $\beta \mathrm{R}=25$. Thick lines correspond to local fitness maxima for the mutant, whereas thin lines correspond to local fitness minima. The monomorphic singular strategies are denoted by $m_{1}{ }^{*}, m_{2}{ }^{*}$ and $m_{3}{ }^{*}$.

As long as the population stays well inside the area of coexistence and away from isoclines, we always have pattern (i), that is, a successfully invading mutant will just oust the resident to which it is most similar after which the population remains dimorphic. Close to an isocline, however, we may also have pattern (ii), that is, the mutant and both former resident types may coexist so that the population becomes trimorphic, at least till the next successful mutant comes along. To understand this we can do a thought experiment in which $m_{2}$ is fixed and does not evolve. The corresponding point on the $m_{1}$-isocline then can be considered as a singular strategy for $m_{1}$ in a population in which $m_{2}$ is now merely an environmental parameter. This means that we can study the fate of $m_{1}$-mutants near the isocline in the same manner as we studied the fate of mutants near a singular strategy in a monomorphic resident population. We thus find that mutual invasibility of a mutant and its resident progenitor in a dimorphic resident population is only possible in the neighbourhood of isoclines. Evolutionary branching may occur if the isocline corresponds to a local fitness minimum, but is unlikely to persevere if evolution in the other resident type moves the population away from the vicinity of the isocline. Near the intersection of two isoclines where directional selection has ceased for both resident types at the same time, however, mutual invasibility will lead to evolutionary branching if at the point of intersection either or both isoclines correspond to local fitness minima.

The points of intersection of isoclines are so-called evolutionarily singular coalitions (Metz et al., 1995; Geritz et al., 1997a,b). Due to the diagonal symmetry of the area of coexistence, singular coalitions always occur in reciprocal pairs, one on each side of the diagonal (Figure 8). Both represent the same population, that is, with the same seed sizes, but with a different order of numbering the resident strategies. A singular coalition is evolutionarily stable (i.e., no mutant can invade) if at the point of intersection both isoclines correspond to local fitness maxima (Vincent and Brown, 1988). Generalization of convergence stability is less straightforward because of the 
(a)

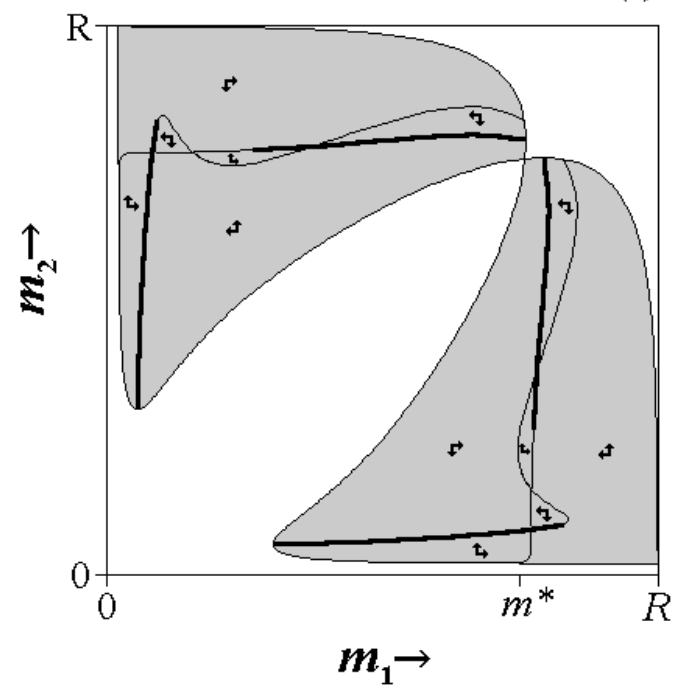

(b)

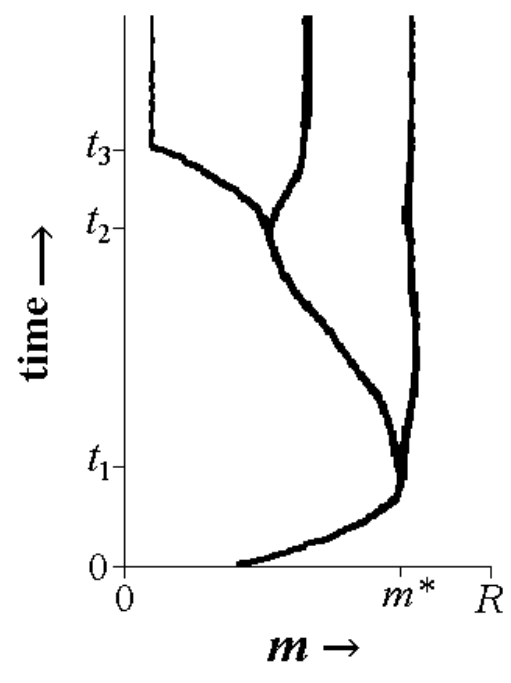

Figure 9. (a) Area of coexistence with isoclines and invasion cones for $\alpha R=9.5$ and $\beta R=35$, and (b) simulated evolutionary tree. At time $t_{1}$ the population reaches the branching point, $m^{*}$, and undergoes evolutionary branching. At time $t_{2}$ the dimorphic population reaches the dimorphic singularity where it for a second time undergoes disruptive selection. At $t_{3}$ the population reaches an evolutionarily stable trimorphism.

higher degree of freedom of evolutionary change in populations with two or more resident strategies. Unlike convergence in monomorphic populations, convergence at higher levels of polymorphism may depend on the relative mutation rates and step sizes of the different resident strategies (Abrams et al., 1993). Unambiguous examples of convergence in dimorphic populations, however, can sometimes be constructed graphically using the invasion cones (Matessi and Di Pasquale, 1996). Like in the monomorphic case, evolutionary stability and convergence stability of dimorphic singular coalitions are fully independent properties that can occur in any combination. A singular coalition that is convergence stable but not evolutionarily stable for at least one of the resident types gives rise to disruptive selection and further branching of the evolutionary tree.

For $\alpha R=6$ and $\beta R=25$ there is a single evolutionarily singular coalition (Figure 8). It can be seen from the invasion cones that the singular coalition will be reached from most places inside the area of coexistence, and therefore is convergence stable. In particular, an initially monomorphic population that enters the area of coexistence near the point $\left(m_{3}^{*}, m_{3}{ }^{*}\right)$ inevitably will end up near the singular coalition. The singular coalition is evolutionarily stable, because at the point of intersection both isoclines represent local fitness maxima. The singular coalition thus represents a final stop of the evolutionary process. The corresponding simulated evolutionary tree is given in Figure 4b.

Figure 9a gives the area of coexistence with isoclines and invasion cones for $\alpha R=9.5$ and $\beta R=35$. Now there are three different evolutionarily singular coalitions (we consider the singularities on either side of the main diagonal as identical). None of these is evolutionarily stable, because at each intersection point one or both isoclines correspond to fitness minima. From the invasion cones it can be seen that the middle of 

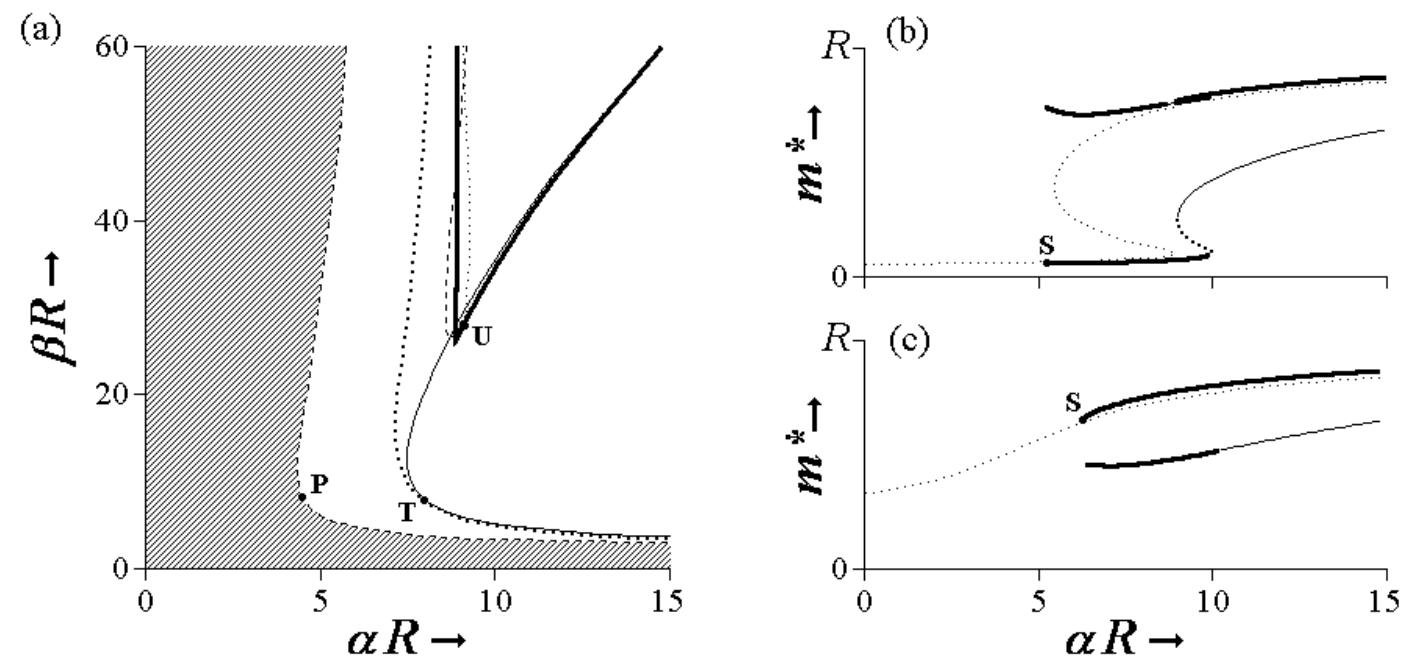

Figure 10. (a) Number and stability properties of the dimorphic singular coalitions as a function of $\alpha R$ and $\beta R$. (b) The actual values of the dimorphic singularities as functions of $\alpha R$ for fixed $\beta R=35$, and (c) $\beta R=5$. The thin dotted curves in (b) and (c) indicate the monomorphic singularities. (See text for further explanation.)

the three singular coalitions is an evolutionary repeller, whereas the other two are convergence stable. For an initially monomorphic population that undergoes evolutionary branching and enters the area of coexistence near the point, $\left(m^{*}, m^{*}\right)$, the probability of reaching the attractor furthest away from the diagonal is negligibly small, as it would require mutations to occur systematically much more frequently in one resident strategy than in the other. Instead, the population evolves towards the singular coalition that is closest to the diagonal. Lacking evolutionary stability, the population then for a second time undergoes disruptive selection and evolutionary branching. The corresponding simulated evolutionary tree is given in Figure 9b. A dimorphic evolutionary attractor that lacks evolutionary stability we shall refer to as a dimorphic branching point.

\section{Change in number and stability properties of evolutionary singular coalitions}

Figure 10a shows how the number and stability properties of the singular coalitions in a dimorphic resident population depend on $\alpha R$ and $\beta R$. Dimorphic singularities do not exist for parameter values inside the hatched region. The border of this region exactly coincides with the boundary between local and global stability of the monomorphic ESS (dotted line in Figure 7a). In the Appendix we show that this is not a coincidence and explain why this should be so. For a population settled at the monomorphic ESS, loss of global evolutionary stability has no consequences if evolution proceeds by small steps only, and the potentially invading mutants are too dissimilar from the resident type to be generated by a single mutation. The new dimorphism, therefore, may remain unreachable until the monomorphic ESS loses also its local evolutionary stability and becomes a branching point at some higher value of $\alpha R$. Only at point "P" where the monomorphic ESS loses its global and local evolutionary stability and turns into a 
branching point all at the same time (Figure 7a), the dimorphic singularity appears just when the ESS becomes a branching point.

For parameter values between the hatched region and the heavy dotted line, there is a single convergence stable and globally evolutionarily stable dimorphic singular coalition. On the other side of the dotted line, the dimorphism has lost its global evolutionary stability but retains its local evolutionary stability. (The dotted line also corresponds to the first occurrence of an evolutionarily stable trimorphism.) The thin solid line indicates where the smaller seed size of the dimorphism loses its evolutionary stability and becomes a local fitness minimum that may give rise to evolutionary branching. At point "T" where the dotted line and the thin solid line touch one another, the population loses both its global and local evolutionary stability simultaneously. At the hatched line (which partly lies inside the wedge-shaped domain formed by the thick solid lines, and which smoothly connects to the right edge of the wedge at point "U"), the larger seed size of the coalition loses its evolutionary stability. Disruptive selection and the ensuing evolutionary branching thus may occur in either the left or the right branch of the evolutionary tree, or both, depending on the exact values of $\alpha R$ and $\beta R$.

The left edge of the wedge-shaped domain corresponds to a fold bifurcation that gives rise to two new singularities when the isoclines first touch and then intersect one another at two additional points. The two new singularities thus formed are respectively a convergence stable but evolutionarily unstable singular coalition and an evolutionary repeller. Inside the parameter range shown, the lack of evolutionary stability of the new attractor is due to the smaller seed size representing a local fitness minimum. The larger seed size of the attractor can also turn into a fitness minimum, but only so at much higher values of $\beta R$ outside the range shown in the figure. The light dotted line inside the wedge (which smoothly connects to the right edge of the wedge at point "U") shows where the larger seed size of the repeller becomes a fitness minimum. The smaller seed size of the repeller always corresponds to a fitness minimum. The right edge of the wedge indicates a fold bifurcation where the repeller and the former evolutionarily stable singular coalition merge and disappear.

Figure $10 \mathrm{~b}$ is a horizontal cross-section through the wedge-shaped domain at $\beta R=35$ showing the actual values of the dimorphic singular coalitions as functions of $\alpha R$. For the sake of comparison, the monomorphic singular strategies have been indicated by a thin dotted line (cf. Figure 7b). The more heavy curves denote the dimorphic singular coalitions. The lower curve indicates the smaller seed size of the coalition, and the upper curve the larger seed size. Thick solid lines correspond to local fitness maxima, thin solid lines to local fitness minima, and the dashed lines indicate evolutionary repellers. A singular coalition is evolutionarily stable only where both curves are thick and solid. The double fold lies exactly in between the edges of the wedge-shaped domain in Figure 10a. The flatness of the fold in the upper curve is directly related to the fact that the isocline for the larger seed size is almost a straight line parallel to the horizontal axis of the coexistence plot (cf. Figure 9a). As $\alpha R$ decreases towards point "S" where the dimorphism becomes extinct and the monomorphic singularity changes from a local ESS on the right side of "S" into a global ESS on the left, the frequency of the larger seed size of the dimorphism gradually becomes zero whereas the smaller seed size converges to the monomorphic ESS 
indicated by the thin dotted line (Figure 10b). In cross-sections through Figure 10a below point "P", however, it is always the smaller seed size that goes extinct while the larger seed size connects to the monomorphic ESS (Figure 10c). In cross-sections exactly through point "P" where the monomorphic singularity loses both its global and its local evolutionary stability and becomes a branching point all at the same time, both seed sizes of the dimorphism connect to the monomorphism.

\section{Generalization to higher levels of polymorphism}

Graphical analyses using pairwise invasibility plots and isoclines is no longer possible for resident populations with three or more coexisting phenotypes. Generalization of the algebraic approach to higher levels of polymorphisms (including the problems with convergence stability) is straightforward (Metz et al., 1995; Geritz et al., 1997a). The fitness, $W_{m 1, \ldots, m \mathrm{n}}\left(m^{\prime}\right)$, of an initially rare mutant with seed size $m^{\prime}$ in a resident population with seed sizes $m_{1}, \ldots, m_{n}$ at its demographic equilibrium directly follows from Equation (12) but with $n$ resident types instead of two. Demographic stability and uniqueness of the resident population equilibrium was verified by simulation of the resident population dynamics using Equation (6). With small evolutionary steps, the direction of evolutionary change in each resident seed size is given by the sign of the corresponding local fitness gradient (cf. Equation 13). The isoclines, where the local fitness gradient is zero, are no longer lines but (n-1)-dimensional manifolds. An evolutionarily singular coalition is a protected polymorphism such that the local fitness gradient is zero for all resident types at the same time (i.e., where all isoclines intersect), and can be found using standard numerical methods for solving systems of nonlinear equations. A singular coalition is evolutionarily stable if at the singular coalition the mutant's fitness as a function of its own strategy has a local maximum at each of the resident strategies (cf. Equation 14).

Figure 11 shows that changes in the number and stability properties of the singular coalitions due to changes in $\alpha R$ and $\beta R$ exhibit similar patterns at all levels of polymorphism. Loss of global evolutionary stability always coincides with the emergence of an evolutionarily stable singularity at a higher level of polymorphism (dotted lines in Figure 11). For parameter values inside the regions numbered "II" through "VI" (separated from one another by the dotted lines) there exist globally evolutionarily stable singular strategies or singular coalitions with, respectively, one up to six different coexisting seed sizes. (Higher levels of polymorphism occur at larger values of $\alpha R$.) On crossing the border between two such regions from the left to the right, the globally evolutionarily stable singular strategy or coalition on the left side loses its global stability and becomes only locally evolutionarily stable. At the same time a new globally evolutionarily stable singular coalition appears consisting of one more resident type (see Appendix). Local evolutionary stability, however, can be lost in various ways, depending on which of the resident seed sizes of the coalition turns into a local fitness minimum first (not shown in Figure 11, but see Figure 10a for loss of local evolutionary stability of dimorphic singularities). At each level of polymorphism there is a wedge-shaped parameter region the edges of which correspond to a fold bifurcation where two singularities simultaneously appear or disappear (thick solid lines in Figure 11). The left edge of each wedge has a vertical asymptote, while the right edge 


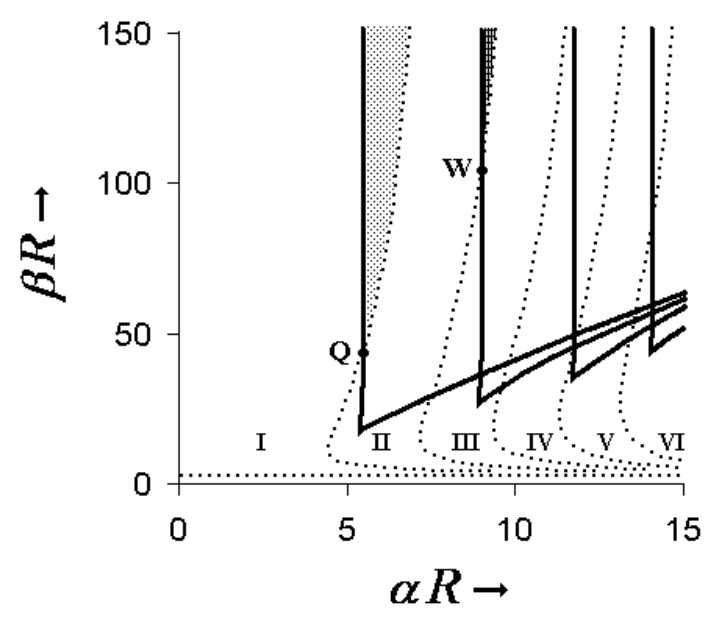

Figure 11. Different levels of evolutionarily stable polymorphisms inside the regions "I" through "VI" separated by the dotted lines. Thick lines indicate fold bifurcations for singularities with one through four seed types present. Notice the different vertical scale compared to Figures $7 a$ and $10 a$. (See text for further explanation.)

converges to a straight line with a positive slope that is the same for all levels of polymorphism. Inside the hatched region near the bottom of the Figure 11, there are no evolutionary singularities.

\section{Extinction of evolutionary branches}

For parameter combinations inside the dotted region in Figure 11 (which is formed by the intersection at point "Q" of the left most wedge shaped domain and region "I"), there are three monomorphic singularities (i.e., an ESS, a repeller and a branching point) but no singularities with two or more coexisting seed types. Figure 12a shows the area of coexistence with isoclines and invasion cones for $\alpha R=6$ and $\beta R=100$, which is well inside this parameter region. Since the isoclines inside the area of coexistence do not intersect, there are no dimorphic singularities. An initially monomorphic population starting inside the domain of attraction of the branching point, $m_{3}{ }^{*}$, will undergo evolutionary branching and will enter the area of coexistence near the point $\left(m_{3}{ }^{*}, m_{3}{ }^{*}\right)$. There being no dimorphic singularity, however, the population evolves along the thick isocline until it reaches the edge of the area of coexistence. Near the edge, the next successful mutant will move the population outside the area of coexistence, so that the population falls back again to a monomorphic condition close to, and inside the domain of attraction of the ESS $m_{1}$ *. This corresponds to the extinction of one of the branches of the evolutionary tree and was described as invasion pattern (iii) at the beginning of this main section. The corresponding simulated evolutionary tree is given in Figure 12b. (N.B., extinctions are also possible for parameter combinations inside a narrow strip along the outside of the right edge of the dotted region in Figure 11, but is too narrow to be seen. For parameter values inside this strip there actually does exist a dimorphic evolutionarily stable singular coalition, but it lies inside a part of the area of coexistence that is unconnected to the part entered by the population during branching, and therefore cannot be reached.) 
(a)

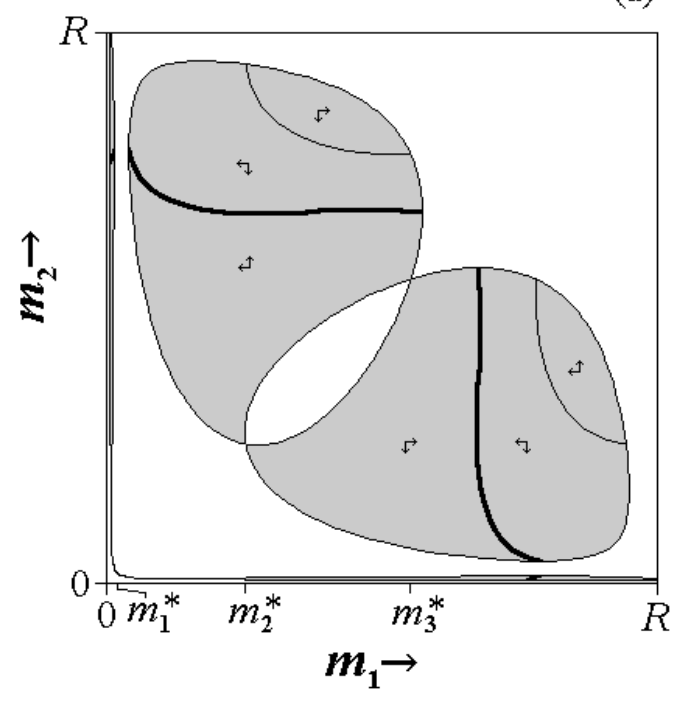

(b)

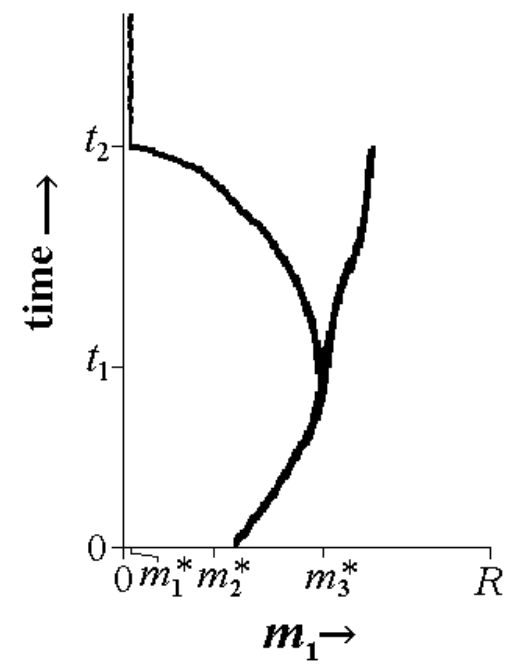

Figure 12. (a) Area of coexistence with isoclines and invasion cones for $\alpha R=6$ and $\beta R=100$, and (b) corresponding simulated evolutionary tree. The initially monomorphic population starting inside the domain of attraction of $m_{3}{ }^{*}$ undergoes evolutionary branching and enters the area of coexistence at time $t_{1}$. The population reaches the edge of the area of coexistence and falls back to a monomorphic state somewhere inside the domain of attraction of $m_{1}^{*}$ at time $t_{2}$.

Extinction is also possible at a higher level of polymorphism. For example, for parameter combinations inside the cross-hatched region in Figure 11 (which is formed by the intersection at point "W" of the second wedge shaped domain and region "II"), there are in addition to the three monomorphic singular strategies also a dimorphic evolutionarily stable singular coalition, a dimorphic attractor lacking evolutionary stability (i.e., a dimorphic branching point) and a dimorphic repeller separating the two. There are no singularities with three or more coexisting resident types, however. An initially monomorphic population starting inside the domain of attraction of the branching point will undergo evolutionary branching twice, that is, at the monomorphic branching point, and next inside the area of coexistence near the dimorphic branching point. The dimorphic evolutionarily stable singular coalition is virtually unreachable because of the repeller in between (cf. Figure 9a). As there are no trimorphic singularities, the population inevitably will fall back again to a dimorphic condition as it reaches the edge of the volume of protected trimorphisms. The new dimorphic coalition, however, is on the other side of the repeller and inside the domain of attraction of the evolutionarily stable dimorphism. A corresponding example of a simulated evolutionary tree for $\alpha R=9.2$ and $\beta R=150$ is given in Figure 13a.

As $\beta R$ increases, there are more and more parts of the parameter space corresponding to different extinction patterns, including cascades of extinctions where a population may drop several levels of polymorphism in succession within a very short time (i.e., 'mass-extinctions). For example, at $\alpha R=9$ and $\beta R=925$ (which is outside the parameter range shown in Figure 11, however), the right edge of region "I" enters the second wedge-shaped domain (not shown), thus forming a parameter region inside the cross-hatched region in which the dimorphic evolutionarily stable singular coalition is missing. After two rounds of evolutionary branching and one extinction event, the 

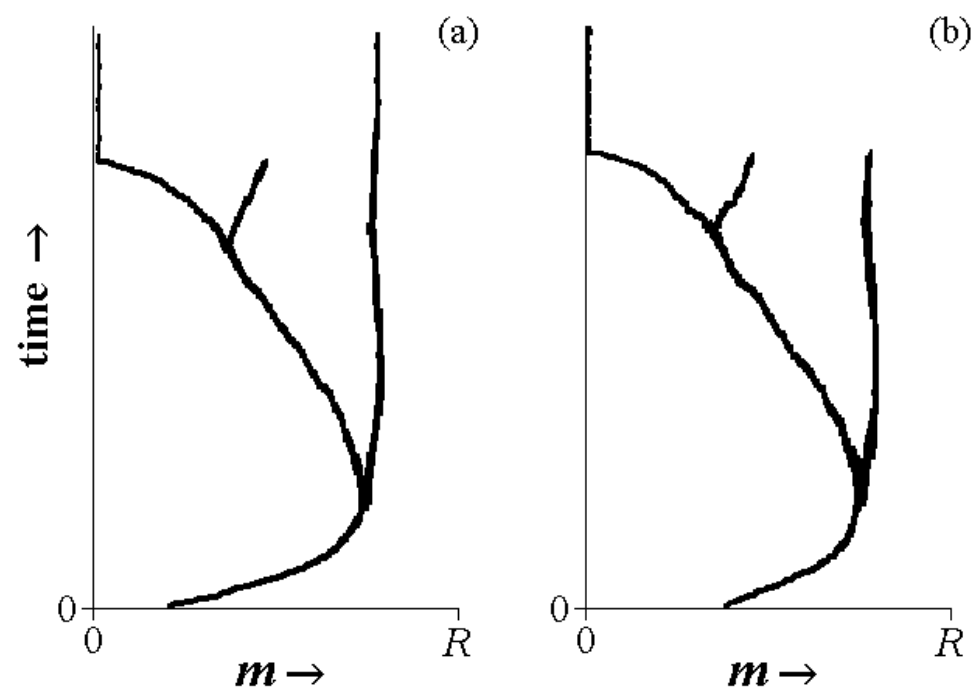

Figure 13. Simulated trees with different extinction patterns for (a) $\alpha R=9.2$ and $\beta R=150$ giving rise to one branch that goes extinct, and (b) $\alpha R=9.04$ and $\beta R=1100$ with two extinct branches.

population will leave the volume of protected trimorphisms and finds itself inside the area of coexistence again, but on the other side of the repeller. However, unlike the previous example, there now is no evolutionarily stable dimorphic coalition, nor can the population return to the former attractor because of the repeller in between. Instead, the population will evolve to the edge of the area of coexistence where it will fall back to a monomorphic condition inside the domain of attraction of the monomorphic ESS. The population thus experiences two branching and two extinction events. The corresponding simulated evolutionary tree for $\alpha R=9.04$ and $\beta R=1100$ is given in Figure $13 b$.

\section{Conclusions and Discussion}

In this paper we investigated how competitive asymmetry due to differences in seed size affects the evolution of seed size under a number of different circumstances. We found that strong competitive asymmetry $(\alpha)$, high resource levels $(R)$ and intermediate harshness of the precompetitive environment $(\beta)$ favor the evolution of variation in seed size.

Varying competitive asymmetry while the amount of resources per germination site and the precompetitive environment remain constant amounts to moving along a horizontal line through the parameter space in Figure 11. If competitive asymmetry is weak, seedlings from large seeds have but a small competitive advantage compared to seedlings from smaller seeds. Consequently, differences in fitness are mainly due to differences in survival during the precompetitive phase of frequency-independent selection rather than to competition, so that, like in the model of Smith and Fretwell (1974), there is only a single evolutionarily stable seed size. As competitive asymmetry increases, evolutionarily stable seed size polymorphisms with progressively larger numbers of different seed sizes become possible. Each such new polymorphism first appears when the former one loses its global evolutionary stability, that is, when it can 
be invaded by mutants sufficiently different from the types already present in the population. Local evolutionary stability is not lost, however, until at some higher degree of competitive asymmetry mutants arbitrarily nearby can also invade. Thus, if only mutants with small phenotypic effect are possible, various evolutionarily stable strategies or coalitions can coexist at the same parameter values. In the limit of extremely asymmetric competition when only the seedling from the single largest seed present in a given site obtains resources while other seedlings from smaller seeds get none, there is an evolutionarily stable seed polymorphism with a contintuous range of seed sizes (Geritz, 1995). Strong competitive asymmetry favors coexistence of alternative strategies, because the competitive advantage of plants with large seeds is sufficiently strong to compensate for their lower per capita seed number, while plants with small seeds have sufficiently many seeds to exploit sites that by chance remain unoccupied by the larger seeds of their superior competitors.

Increasing or decreasing the total amount of resources per germination site while the competitive asymmetry and the precompetitive environment remain constant amounts to moving up or down along a straight line through the origin in Figure 11. The slope of this line is equal to the ratio $\beta: \alpha$. A large ratio corresponds to large seeds having either only a minor precompetitive advantage relative to most smaller seeds except for the smallest (large $\beta$; cf. Figure $2 \mathrm{a}$ ), or a small advantage during competiton (small $\alpha$ ) or both. Varying the amount of resources has very much the same effect as changing the competitive asymmetry: If resources are scarce, the population density is too low for competition to be a significant evolutionary factor. Consequently, frequencyindependent selection during the precompetitive phase dominates, so that there is only a single evolutionarily stable seed size. As the amount of resources increases, evolutionarily stable polymorphisms with progressively larger numbers of coexisting seed sizes become possible. However, if the slope of the line is sufficiently steep (i.e., steeper than the asymptotic slope of the right edge of the wedge-shaped domain in Figure 6), then the monomorphic ESS retains its (local) evolutionary stability and exists next to higher levels of evolutionarily stable seed polymorphism irrespectively of the amount of resources.

Changing only the precompetitive environment while the competitive asymmetry and the amount of resources per germination site remain constant amounts to moving along a vertical line in Figure 11. The highest possible level of evolutionarily stable seed polymorphism is reached at intermediate types of the precompetitive environment when seedling establishment is moderately low but large seeds have a significant precompetitive advantage compared to smaller seeds. If seedling establishment becomes too low, however, surviving the precompetitive phase of frequency-independent selection determines the outcome of evolution, so that we only find a single evolutionarily stable seed size. If, on the other hand, seedling establishment is high for small and large seeds alike, small seeds tend to be favored, because small seeds can be produced in larger numbers and no longer have a precompetitive disadvantage compared to larger seeds.

Assuming mutation-limited evolution and small evolutionary steps, an initially monomorphic population can reach an evolutionarily stable dimorphism only by means of evolutionary branching during which the population undergoes disruptive selection 
and gives rise to two subsequently phenotypically diverging daughter lineages each with its own seed size. Higher levels of polymorphism are reached through a series of successive branching events interspersed by stretches of directional selection. Evolutionary branching occurs only near evolutionary singularities that are convergence stable but lack evolutionary stability, called branching points (Metz et al., 1995; Geritz et al., 1997a,b). When more than one evolutionarily stable outcome is possible, it depends on the initial resident seed sizes to which the population will actually evolve (cf. Figure 4). If precompetitive survival of seeds and seedlings is high for both small and large seeds (large $\beta$ ), evolutionary branching can be followed by the extinction of one or more lineages (including the possibility of mass-extinction). In the long run, therefore, evolutionary branching does not always lead to higher levels of seed polymorphisms (cf. Figures $12 \mathrm{~b}$ and 13).

Whereas the branching points determine the number of branches of the evolutionary tree, other factors may have an effect on the precise shape of the evolutionary tree. First, at a branching point (like at any evolutionary singularity), the local fitness gradient is zero, so that fitness differences of nearby mutants are necessarily very small. Advantageous mutants can therefore spread only slowly and remain rare for relatively long periods. While rare, they are a poor source of new mutants necessary for further evolution of the population, and have moreover a high probability of becoming extinct due to genetic drift. Evolutionary branching, therefore, is a slow process relative to evolution during the stretches of directional selection (Figures $4 \mathrm{~b}$ and $9 \mathrm{~b}$ ). Genetic drift was not accounted for in the present simulations of the evolutionary tree, so that the difference between the two phases is less pronounced than it would have been in stochastic individual-based simulations. Second, small seeds can be produced in larger numbers and plants with small seeds tend to reach higher densities than plants with larger seeds. Consequently, with a constant mutation probability per offspring produced, the total mutation frequency increases as seeds become smaller, so that evolution accelerates towards smaller seed sizes (Figures $4 b$ and 9b). Third, in Figure 9a, the dimorphic population evolves along the isocline of the larger seed size, so that the shape of the isocline determines the shape of the evolutionary trajectory. The relative constancy of the rightmost branch of the evolutionary tree in Figure $9 \mathrm{~b}$ is therefore due to the isocline being almost a straight line perpendicular to the corresponding axis.

Below we consider the significance of some of the model assumptions. Under the present assumptions where all offspring have the same seed size as the mother, the model only applies to apomictic species or to species that are strictly selfing. In order to apply the results more generally to outbreeding species as well, we must assume that there occurs no hybridization between the different branches of the evolutionary tree, so that different lineages breed true. Geritz et al. (1997) argue that if hybrid offspring have intermediate phenotypes, then evolutionary branching may favor the evolution of reproductive isolation, because during branching intermediate phenotypes have low fitness. Specific models support this view (Kisdi and Geritz, in prep.; Doebeli and Dieckmann, in prep.).

In the model, each plant type produces seeds of one size only. Thus, by assumption, any variation in seed size in a population is due to differences between plants rather than within plants. The evolutionarily stable outcomes of the model, 
however, can be interpreted more generally as the result of different plant types producing seeds of one specific size each, or of one plant type producing seeds of different sizes in the corresponding frequencies, or any combination of these two (Geritz, 1995). The corresponding evolutionary dynamics (as opposed to the final evolutionary outcomes) will generally not be the same, however. The adaptive growth and branching of the evolutionary tree modeled here, therefore, applies only if each plant type has its own specific seed size.

Although the model was formulated for annual plants and for germination sites all with the same amount of resources, Geritz (1995) showed that with extremely asymmetric competition the same results hold for perennials if $R$ is replaced by the expected total amount of resources that becomes available in a site during a plant's lifetime (also, see Geritz et al. 1988), and for varying amounts of resources among different sites if $R$ is replaced by its population average. The arguments developed there directly generalize to intermediate degrees of competitive asymmetry.

In order to compare predicted evolutionarily stable seed size distributions with observed seed size distributions in real plant populations, competitive asymmetry should somehow be measured. First, notice from Equation (3) that the ratio of the competitive abilities of the various plants inside a given germination site is equal to the ratio of the resources each individual plant obtains. This is due to the denominator of Equation (3) being the same for all plants present in a given site. We thus have

$$
\ln c_{1}-\ln c_{2}=\ln R_{1}-\ln R_{2},
$$

where $c_{1}, c_{2}$ and $R_{1}, R_{2}$ are respectively the competitive abilities and the amount of resources obtained by two arbitrary plants chosen from the same germination site. Second, notice from Equation (4) that the competitive asymmetry, $\alpha$, is equal to the proportional increase in competitive ability due to an increase in seed size or, equivalently,

$$
\alpha=\frac{d \ln c}{d m} .
$$

Combining Equations (15) and (16), it follows that competitive asymmetry can be estimated by

$$
\alpha_{e s t}=\frac{\ln R_{1}-\ln R_{2}}{m_{1}-m_{2}},
$$

where $m_{1}$ and $m_{2}$ denote the size of the respective seeds from which the two plants have emerged. The quantities $R_{1}$ and $R_{2}$ can in turn be estimated by the total per capita seed weight or by the total plant dry weight. The estimated competitive asymmetry should be approximately the same for any two plants selected from the same germination site, and should moreover be independent of both the local plant density (i.e., the number of competitors inside the germination site) and the local amount of resources. Notice that $\alpha_{e s t}$ describes the potential competitive advantage of a larger seed size independently of the actual competition intensity, and differs in this respect from other frequently used measures of plant competition intensity or hierarchy in plant size induced by competition (Benjamin, 1988; Grace, 1995). 
We are not aware of any empirical data that directly supports the particular functional form relating seed size to competitive ability. The reason why we have used the form given in Equation (4) is that it has the desired property that plants from larger seeds are better competitors and have a disproportionately large advantage over plants from smaller seeds (Stanton, 1985; Wulff, 1986b). A less realistic property, however, may be that in the model competitive asymmetry could become arbitrarily large for sufficiently large differences in seed size. In reality, relative germination date and plant spacing, which were not included in the model, also affect plant competition (Black and Wilkinson, 1963; Ross and Harper, 1972; Ford and Diggle, 1981; Howell, 1981), and may effectively set an upper limit to the maximum possible degree of competitive asymmetry due to differences in seed size.

If competition is asymmetric, an initial advantage of one individual over its neighbors is accentuated during subsequent growth, so that the distribution of resources among different plants becomes increasingly uneven towards the end of the season. If the growing season is short, individual differences will be small, while if it is long, differences will be more pronounced. The potential advantage of a larger seed size thus may depend on the length of the growing season. Season length decreases with global latitude, which implies that competitive asymmetry is likely to be stronger near the tropics than at higher latitudes. Since in the model strong competitive asymmetry favors the coexistence of plants with different seed sizes, we hypothesize that on a global scale seed size variation should decrease with latitude such that near the arctic only small seeds remain. Likewise, the model predicts a systematic decline of seed size variation from the base towards the top of a mountain. Moreover, a change in season length due to a change in climate may cause competitive asymmetry to vary on a geological timescale. Thus, during periods of global warming the model predicts an increase in seed size variation, while during periods of glaciation we expect less variation.

Most models of competitive asymmetry in plants address the issue of selfthinning and the emerging size-hierarchy in plant populations (Aikman and Watkinson, 1980; Ford and Diggle, 1981; Benjamin, 1988; Bonan, 1988), or the issue of biodiversity and limiting similarity (Fagerström and Ågren 1979; Tilman 1994; Abrams 1996). In an evolutionary context, Rees and Westoby (1997) consider the possibility of evolutionarily stable coalitions of plants with different seed sizes depending on the strength of competitive asymmetry. Although in their model they use a different approach and do not explicitly consider long-term evolutionary dynamics, their results are largely consistent with ours. Matsuda and Abrams (1994) argue that strong competitive asymmetry within a species can lead to runaway evolution to selfextinction. Because large trait values have an associated cost (such as, e.g., a lower per capita seed number in the present model), runaway evolution is likely to result in smaller populations. Although in deterministic models populations may never actually reach zero density, in reality they may become sufficiently small that eventually stochastic factors cause their extinction. In the present model we assume populations to be always large enough for demographic stochasticity to be negligible. However, with very strong competitive asymmetry (large $\alpha$ ), this assumption may no longer be attainable. At a high degrees of competitive asymmetry, seed size must first become very large (with corresponding low seed numbers and low population density) before it could undergo evolutionary branching (cf. Figure $7 b, c)$. Demographic stochasticity then 
can no longer be ignored and may drive the population to extinction before it reaches the branching point. Self-extinction may in turn lead to taxon cycles if, on a larger timescale, the habitat is periodically recolonized by seeds produced elsewhere. Recolonization then is most probably due to small seeds, because they are produced in larger numbers, and tend to have a higher dispersibility. (For related examples of taxon cycles, see, e.g., Maynard Smith and Brown, 1986; Taper and Case, 1992; Abrams and Matsuda, 1994; Matsuda and Abrams, 1994.) Evolutionary cycles found by Law et al. (1997) in a model with competitive asymmetry are of a different type and are driven by differences in competitive asymmetry within species, and are not possible in the present model.

Various results presented here are model-independent and point the way to a more general evolutionary bifurcation theory describing how the number and stability properties of evolutionary singularities change as a consequence of changes in model parameters. Such evolutionary bifurcation theory differs from classical bifurcation theory (e.g., Kuznetsov, 1995) in that more than one stability concept is involved, that is, evolutionary stability and convergence stability. Geritz et al. (1997a,b) also consider two additional stability concepts concerning the invading capability of the singular strategy and the possibility of nearby polymorphisms. Bifurcation of evolutionary singularities, therefore, may yield both novel and potentially more complex patterns than in the classical theory. For example, we showed that singular strategies can annihilate one another (or, equivalently, can simultaneously appear if the parameter is changed in the opposite direction) either as a pair of a non-ESS repeller and a branching point, or as a pair of a convergence stable ESS and an ESS that lacks convergence stability. The first possibility is illustrated for the present model in Figure 6, and shows that an ESS necessarily must lose its evolutionary stability before it can disappear by merging with an evolutionary repeller. The second possibility (which does not occur in the model) arises if the pattern of shading in Figure 6 is reversed. Moreover, in the Appendix we show that isoclines connect to the boundary of the set of protected polymorphisms only at specific places, and that as a consequence there is an intimate relation between evolutionary singularities on different levels of polymorphism. For example, in the present model loss of global evolutionary stability always coincides with the emergence of an evolutionarily stable singularity on a higher level of polymorphism. 


\section{Appendix}

\section{Isoclines and their connection to the boundary of the area of coexistence}

Isoclines connect to the boundary of the area of coexistence only at specific places (Metz et al., 1996). These places depend on the positions of the monomorphic singularities and on the shape of the area of coexistence. Moreover, the direction of the fitness gradient on either side of the isocline close to its connection to the boundary, and also whether the isocline corresponds to a local fitness maximum or fitness minimum, too, follow from the monomorphic singularities and the shape of the area of coexistence. As a consequence, there is an intimate relationship between monomorphic and dimorphic singularities that can be used to understand various outcomes of the present model. The theory given below applies more generally, however, and is not just confined to the present model. Moreover, similar arguments apply to higher levels of polymorpisms as well, although a graphical representation becomes troublesome.

Figure 14 shows the area of coexistence for $\alpha R=6$ and $\beta R=25$ with the $m_{1}$ isoclines and arrows that indicate the direction of evolution in $m_{1}$. (We here only consider the $m_{1}$-isocline, because the $m_{2}$-isocline is obtained by simply taking the mirror image of the $m_{1}$-isocline along the main diagonal.) The boundary of the area of coexistence is made up of two curves defined by $W_{m 1}\left(m_{2}\right)=1$ or $W_{m 2}\left(m_{1}\right)=1$. The $m_{1}$ isocline connects to segments of the boundary defined by $W_{m 1}\left(m_{2}\right)=1$ at any point that is vertically above a singular strategy on $m_{1}$-axis (Figure 14 ; solid circles). The reason for this can be seen intuitively as follows. Inside the area of coexistence $m_{2}$ is protected against extinction by an annual growth rate that is larger than one when $m_{2}$ happens to be rare. On the boundary where $W_{m 1}\left(m_{2}\right)=1$ this is no longer the case, so that if we approach the boundary from the inside of the area of coexistence, the frequency of $m_{2}$ at the demographic equilibrium gradually dwindles to zero. Near the boundary, therefore, the effect of $m_{2}$ on the evolution of $m_{1}$ is negligible, and the direction of evolution in $m_{1}$ must be the same as if the population were monomorphic. In particular, if $m_{1}$ coincides with a singular strategy in a monomorphic population, then any point on the boundary vertically above $m_{1}$ will also have a fitness gradient that is zero and thus must lie on the $m_{1}$-isocline (Figure 14; solid circles). Moreover, if $m_{1}$ is evolutionarily stable or lacks evolutionary stability, then on the boundary the isocline corresponds also to, respectively, a local fitness maximum (Figure 14; points "q" and "r") or a local fitness minimum (Figure 14; points "o", "p", "tt" and "u").

The $m_{1}$-isocline connects to segments of the boundary defined by $W_{m 2}\left(m_{1}\right)=1$ at any point where the boundary has a horizontal tangent (Figure 14; open circles). The reason for this can be seen intuitively as follows. For a population virtually on the boundary of the area of coexistence, only mutants of $m_{1}$ further into the interior of the area of coexistence can invade, whereas mutations directed towards the outside cannot. In other words, along the boundary, the local fitness gradient with respect to $m_{l}$ points to the inside of the area of coexistence. At each point where the boundary has a horizontal tangent, therefore, the fitness gradient changes direction, so that the point of contact with the tangent must lie on the $m_{1}$-isocline (Figure 14 ; open circles). If at the point of 


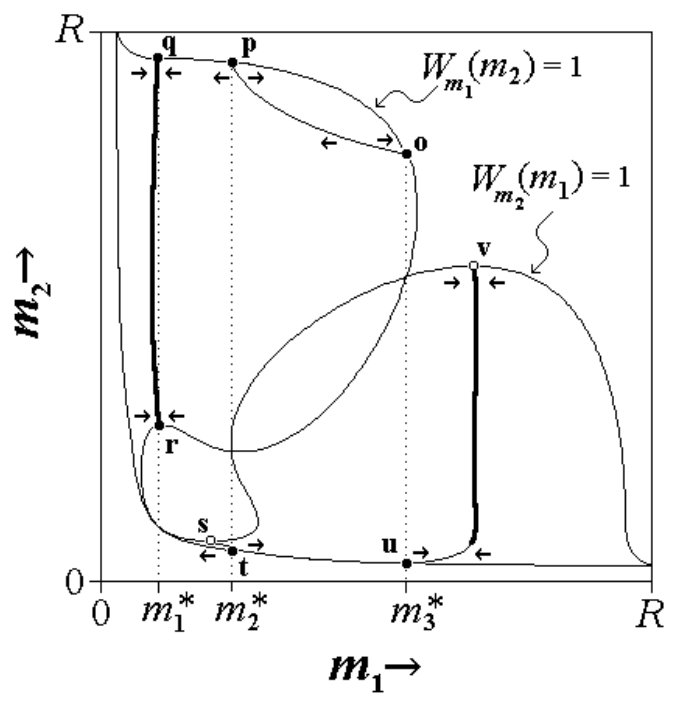

Figure 14. Connections of the $m_{1}$-isocline to the boundary of the area of coexistence for $\alpha R=6$ and $\beta R=25$. The monomorphic ESS, repeller and branching point are denoted by, respectively, $m_{1}{ }^{*}, m_{2}{ }^{*}$ and $m_{3}^{*}$.

contact the boundary is locally convex, the fitness gradient points towards the isocline (Figure 14; point " $\mathbf{v}$ "). Moreover, since any change in $m_{1}$ at the point of contact brings the population outside the area of coexistence, no mutant can invade, and hence the isocline corresponds to a fitness maximum with respect to mutations in $m_{1}$. If the boundary of the area of coexistence at the point of contact is locally concave, however, then the fitness gradient points away from the isocline (Figure 14; point "s"). Moreover, any change in $m_{1}$ at the point of contact brings the population inside the area of coexistence, so that any mutant could invade, and the isocline thus represents a local fitness minimum with respect to mutations in $m_{1}$.

\section{Loss of global evolutionary stability and the emergence of new singularities}

An ESS loses its global evolutionary stability when in the pairwise invasibility plot the set of potential invading mutants extends above the point on the horizontal axis representing the ESS (e.g., Figure 5; $\alpha R=5.0, \beta R=25$ ). For a population already settled at the ESS this has no consequences if evolution proceeds by small steps only and the potentially invading mutants are too dissimilar from the resident type to be generated by a single mutation. However, if the extension of the set of potentially invading mutants also leads to an extension of the area of coexistence, then a new, evolutionarily stable dimorphic singularity appears at the very same moment the ESS loses it global evolutionary stability. This is a direct consequence of the way isoclines are connected to the boundary of the area of coexistence. Similar arguments apply to higher levels of polymorphism although a graphical representation may no longer be possible.

Figure 15 shows the area of coexistence with isoclines and invasion cones before, during and after the ESS, $m^{*}$, loses its global evolutionary stability at, respectively, $\alpha R=4.40,4.45$ and 4.50 , and for fixed $\beta R=15$. When the ESS is still 
(a)

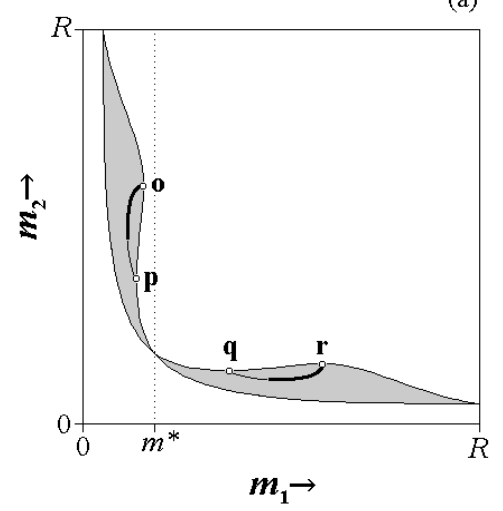

(b)

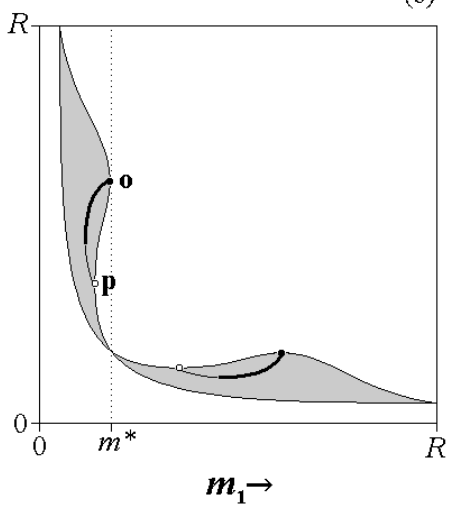

(c)

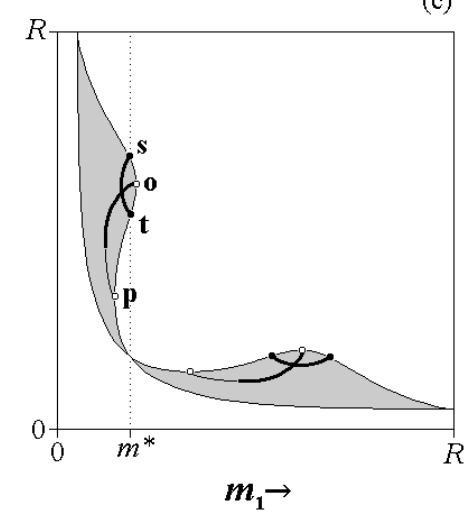

Figure 15. Loss of global evolutionary stability of an ESS and the simultaneous appearance of an evolutionarily stable and convergence stable dimorphic singularity. (a) $\alpha R=4.40$, (b) $\alpha R=4.45$ and (c) $\alpha R=4.50$ and fixed $\beta R=15$. The ESS is denoted by $m^{*}$.

globally evolutionarily stable (Figure 15a), the isoclines do not intersect, and hence there are no dimorphic singularities. The $m_{1}$-isocline connects points "q" and "r" where the boundary of the area of coexistence has horizontal tangents. Its mirror image, the $m_{2^{-}}$ isocline, connects points "o" and "p". When the ESS is about to lose its global evolutionary stability (Figure 15b), point "o" has moved to a position directly above the ESS, and therefore now also coincides with a point on the $m_{1}$-isocline. At this stage, however, the $m_{1}$-isocline exists in a collapsed state consisting only of one point on the boundary. Moreover, as point "o" belongs to both the $m_{1}$-isocline and the $m_{2}$-isocline, it also represents a dimorphic singularity. Once the ESS has lost its global evolutionary stability (Figure 15c), there are two points ("s" and " $\mathbf{t}$ ") on the boundary of the area of coexistence that lie directly above the ESS. The $m_{1}$-isocline is therefore no longer a single point, but has expanded into a curve with endpoints " $\mathbf{s}$ " and " $\mathbf{t}$ ", while at the same time the dimorphic singularity has moved from the boundary into the interior of the area of coexistence. During the initial phase just after the ESS has lost its global evolutionary stability, the dimorphic singularity is necessarily evolutionarily stable, because both the $m_{1}$-isocline (which inherits its stability properties from the ESS) and the $m_{2}$-isocline in the neighborhood of its endpoint "o" (where the area of coexistence is locally convex) correspond to fitness maxima.

The dimorphic singularity in its primordial stage on the boundary of the area of coexistence when the ESS is just about to lose its global evolutionary stability (Figure 15b) consist of two seed types, the smaller of which has the same seed size as the ESS, while the other represents the first of mutants that can invade the ESS at slightly higher values of $\alpha R$, but has still a zero frequency itself. This explains why in Figure $10 \mathrm{~b}$ it is the lower and not the upper branch of the dimorphism that connects to the thin dotted line representing the monomorphic singularities. For values of $\beta R$ below point "P" in Figure 10a, the situation is the reverse, because then the first of mutants that can invade the ESS has a seed size smaller than that of the ESS (Figure 10c). Only at point "P" itself where the ESS loses its global and local evolutionary stability at the same time, the first mutants to invade are on either side and arbitrarily close to the former ESS, so that in its primordial stage both seed sizes of the dimorphic singularity coincide with the monomorphic singularity. 


\section{References}

Abrams, P. A. (1996) Limits to the similarity of competitors under hierarchical lottery competition. American Naturalist 148, 211-219.

Abrams, P. A., and Matsuda, H. (1994) The evolution of traits that determine ability in competitive contests. Evolutionary Ecology 8, 667-686.

Abrams, P. A., Matsuda, H. and Harada, Y. (1993) Evolutionary unstable fitness maxima and stable fitness minima of continuous traits. Evolutionary Ecology 7, 465-487.

Ågren, G. I. and Fagerström, T. (1984) Limiting dissimilarities in plants: Randomness prevents exclusion of species with similar competitive abilities. Oikos 43, 369375.

Aikman, D. P. and Watkinson, A. R. (1980) A model for growth and self-thinning in even-aged monocultures of plants. Annals of Botany 45, 419-427.

Arnold, S. (1983) Sexual selection: The interface of theory and empiricism. In: P. Bateson (ed.) Mate choice, pp. 67-107, Cambridge University Press, Cambridge.

Bell, G., Lechowicz, M. J. and Schoen, D. J. (1991) The ecology and genetics of fitness in forest plants. III. Environmental variance in natural populations of Impatiens pallida. Journal of Ecology 79, 697-713.

Benjamin, L. R. (1988) A single equation to quantify the hierarchy in plant size induced by competition within monocultures. Annals of Botany 62, 199-214.

Black, J. N. (1958) Competition of plants of different initial seed sizes in swards of subterranean clover (Trifolium subterraneum L.) with particular reference to leaf area and the light microclimate. Australian Journal of Agricultural Research 9, 299-318.

Black, J. N. and Wilkinson, G. N. (1963) The role of time of emergence in determining the growth of individual plants in swards of subterranean clover (Trifolium subterraneum L.). Australian Journal of Agricultural Research 14, 628-638.

Bonan, G. B. (1988) The size structure of theoretical plant populations: Spatial patterns and neighborhood effects. Ecology 69, 1721-1730.

Christiansen, F. B. (1991) On conditions for evolutionary stability for a continuously varying character. American Naturalist 138, 37-50.

Cipollini, M. L. and Stiles, E.W. (1991) Seed predation by the bean weevil Acanthoscelides obtectus on Phaseolus species: consequences for seed size, early growth and reproduction. Oikos 60, 205-214.

Eshel, I. (1983) Evolutionary and continuous stability. Journal of Theoretical Biology 103, 99-111.

Eshel, I. and Motro, U. (1981) Kin selection and strong evolutionary stability of mutual help. Theoretical Population Biology 19, 420-433. 
Eshel, I., Motro, U. and Sansone, E. (1997) Continuous stability and evolutionary convergence. Journal of Theoretical Biology 185, 333-343.

Fagerström, T. and Ågren, G. I. (1979) Theory for coexistence of species differing in regeneration properties. Oikos 33, 1-10.

Fenner, M. (1983) Relationships between seed weight, ash content and seedling growth in twenty-four species of Compositae. New Phytologist 95, 697-706.

Ford, E. D. (1975) Competition and stand structure in some even-aged plant monocultures. Journal of Ecology 63, 311-333.

Ford, E. D. and Diggle, P. J. (1981) Competition for light in a plant monoculture modeled as a spatial stochastic process. Annals of Botany 48, 481-500.

Garciá-Dorado, A. (1990) Some evolutionary properties of parental investment per offspring in a heterogeneous environment. Journal of Theoretical Biology 147, 101-114.

Geritz, S. A. H. (1995) Evolutionarily stable seed polymorphism and small-scale spatial variation in seedling density. American Naturalist 146, 685-707.

Geritz, S. A. H. (1997) Coevolution of seed size and seed predation. Evolutionary Ecology (in press).

Geritz, S. A. H., Kisdi, É., Meszéna, G. and Metz, J. A. J. (1997a) Evolutionarily singular strategies and the adaptive growth and branching of the evolutionary tree. Evolutionary Ecology (in press).

Geritz, S. A. H., Metz, J. A. J., Kisdi, É. and Meszéna, G. (1997b) The dynamics of adaptation and evolutionary branching. Physical Review Letters 78, 2024-2027.

Geritz, S. A. H., Metz, J. A. J., Klinkhamer, P. G. L. and De Jong, T. J. (1988) Competition in safe-sites. Theoretical Population Biology 33, 161-180.

Grace, J. B. (1995) On the measurement of plant competition intensity. Ecology 76, 305-308.

Grime, J. P. and Jeffrey, D. W. (1965) Seedling establishment in vertical gradients of sunlight. Journal of Ecology 53, 621-642.

Gross, K. L. (1984) Effects of seed size and growth form on seedling establishment of six monocarpic perennial plants. Journal of Ecology 72, 369-387.

Gross, K. L. and Werner, P. A. (1982) Colonizing abilities of "biennial" plant species in relation to ground cover: implications for their distributions in a successional sere. Ecology 63, 921-931.

Harper, J. L., Lovell, P. H. and Moore, K. G. (1970) The shapes and sizes of seeds. Annual Review of Ecology and Systematics 1, 327-356.

Haskins, F. A. and Gorz, H. J. (1975) Influence of seed size, planting depth, and companion crop on emergence and vigor of seedlings in sweetclover. Agronomy Journal 67, 652-654. 
Houssard, C. and Escarré, J. (1991) The effects of seed weight on growth and competitive ability of Rumex acetosella from two successional old-fields. Oecologia 86, 236-242.

Howell, N. (1981) The effect of seed size and relative emergence time on fitness in a natural population of Impatiens $\underline{\text { capensis }}$ Meerb. (Balsaminaceae). American Midland Naturalist 105, 312-320.

Jones, M. B. (1978) Aspects of the biology of the big-handed crab, Heterozius rohendifrons (Decapoda: Brachyura), from Kaikoura, New Zealand. New Zealand Journal of Zoology 5, 783-794.

Jurado, E. and Westoby, M. (1992) Seedling growth in relation to seed size among species of arid Australia. Journal of Ecology 80, 407-416.

Kisdi, É. and Meszéna, G. (1993) Density-dependent life history evolution in fluctuating environments. In: C. W. Clark, and J. Yoshimura (eds.). Adaptation in a stochastic environment. Lecture Notes in Biomathematics 98, 26-62.

Kisdi, É. and Meszéna, G. (1995) Life history with lottery competition in a stochastic environment: ESSs which do not prevail. Theoretical Population Biology 47, 191221.

Kuznetsov, Y. A. (1995) Ellements of applied bifurcation theory. Springer-Verlag New York, Inc.

Lalonde, R. G. (1991) Optimal offspring provisioning when resources are not predictable. American Naturalist 138, 680-686.

Law, R., Marrow, P. and Dieckmann, U. (1997) On evolution under asymmetric competition. Evolutionary Ecology 11, 485-501.

Matessi, C. and Di Pasquale, C. (1996) Long term evolution of multi-locus traits. Journal of Mathematical Biology 34, 613-653.

Matsuda, H. and Abrams, P. A. (1994) Runaway evolution to self-extinction under asymmetric competition. Evolution 48, 1764-1772.

Maynard Smith, J. (1982) Evolution and the theory of games, Cambridge University Press, Cambridge.

Maynard Smith, J. and Brown, R. L. W. (1986) Competition and body size. Theoretical Population Biology 30, 166-179.

McGinley, M. A. and Charnov, E. L. (1988) Multiple resources and the optimal balance between size and number of offspring. Evolutionary Ecology 2, 77-84.

McGinley, M. A., Temme, D. H. and Geber, M. A. (1987) Parental investment in offspring in variable environments: Theoretical and empirical considerations. American Naturalist 130, 370-398.

Meszéna, G., Czibula, I. and Geritz, S. A. H. (1997) Adaptive dynamics in a two-patch environment: A toy model for allopatric and parapatric speciation. J. Biol. Sys. (in press). 
Metz, J. A. J., Geritz, S. A. H., Meszéna, G., Jacobs, F. J. A. and Van Heerwaarden, J. S. (1996) Adaptive dynamics: A geometrical study of the consequences of nearly faithful reproduction. In: S. J. van Strien and S. M. Verduyn Lunel (eds.). Stochastic and spatial structures of dynamical systems. North Holland, Elsevier. pp. 183-231.

Metz, J. A. J., Nisbet, R. and Geritz, S. A. H. (1992) How should we define 'fitness' for general ecological scenarios? Trends in Ecology and Evolution 7, 198-202

Michaels, H. J., Benner, B., Hartgerink, A. P., Lee, T. D. and Rice, S. (1988) Seed size variation: magnitude, distribution, and ecological correlates, Evolutionary Ecology 2, 157-166.

Mogie, M., Latham, J. R. and Warman, E. A. (1990) Genotype-independent aspects of seed ecology in Taraxacum. Oikos 59, 175-182.

Osunkoya, O.O., Ash, J. E., Hopkins, M. S. and Graham, A. W. (1994) Influence of seed size and seedling ecological attributes on shade-tolerance of rain-forest tree species in northern Queensland. Journal of Ecology 82, 149-163.

Paris, O. H. and Pitelka, F. A. (1962) Population characteristics of the terrestrial isopod Armadillidium vulgare in California grassland. Ecology 43, 229-248.

Rees, M., and Westoby, M. (1997) Game-theortical evolution of seed mass in multispecies ecological models. Oikos 78, 116-126.

Perrins, C. M. (1965) Population fluctuations and clutch size in the great tit, Parus major L. Journal of Animal Ecology 34, 601-647.

Ross, M. A. and Harper, J. L. (1972) Occupation of biological space during seedling establishment. Journal of Ecology 60, 77-88.

Sakai, S. (1995) A model for seed size variation among plants. Evolutionary Ecology 9, 495-507.

Sakai, S. and Sakai, A. (1995) Flower size-dependent variation in seed size: Theory and a test. American Naturalist 145, 918-934.

Schluter, D. (1988) Estimating the form of natural selection on a quantitative trait. Evolution 42, 849-861.

Smith, C. C. and Fretwell, S. D. (1974) The optimal balance between size and number of offspring. American Naturalist 108, 499-506.

Stanton, M. L. (1984) Seed variation in wild radish: effect of seed size on components of seedling and adult fitness. Ecology 65, 1105-1112.

Stanton, M. L. (1985) Seed size and emergence time within a stand of wild radish (Raphanus raphanistrum L.): the establishment of a fitness hierarchy. Oecologia 67, 524-531.

Taper, M. L. and Case, T. J. (1992) Models of character displacement and the theoretical robustness of taxon cycles. Evolution 46, 317-333.

Taylor, P. D. (1989) Evolutionary stability in one-parameter models under weak selection. Theoretical Population Biology 36, 125-143. 
Temme, D. H. (1986) Seed size variability: A consequence of variable genetic quality among offspring? Evolution 40, 414-417.

Tilman, D. (1994) Competition and biodiversity in spatially structured habitats. Ecology $75,2-16$.

Van Tienderen, P. and De Jong, G. (1986) Sex ratio under the haystack model: Polymorphism may occur. Journal of Theoretical Biology 122, 69-81.

Venable, D. L. (1985) The evolutionary ecology of seed heteromorphism. American Naturalist 126, 577-595.

Vincent, T. L. and Brown, J. S. (1988) The evolution of ESS theory. Annual Review of Ecology and Systematics 19, 423-443.

Weiner, J. (1990) Asymmetric competition in plant populations. Trends in Ecology and Evolution 5, 360-364.

Westoby, M., Jurado, E. and Leishman, M. (1992) Comparative evolutionary ecology of seed size, Trends in Ecology and Evolution 7, 368-372.

Winn, A. A. (1985) Effects of seed size and germination site on seedling emergence of Prunella vulgaris in four habitats. Journal of Ecology 73, 831-840.

Winn, A. A. (1988) Ecological and evolutionary consequences of seed size in Prunella vulgaris. Ecology 69,1537-1544.

Wulff, R. D. (1986a) Seed size variation in Desmodium paniculatum. II. Effects on seedling growth and physiological performance. Journal of Ecology 74, 99-114.

Wulff, R. D. (1986b) Seed size variation in Desmodium paniculatum. III. Effects on reproductive yield and competitive ability. Journal of Ecology 74, 115-121. 